\title{
An Investigation of Spiral Gravity Waves Radiating from Tropical Cyclones Using a Linear, Nonhydrostatic Model
}

\author{
DAVID S. NOLAN \\ Rosenstiel School of Marine and Atmospheric Science, University of Miami, Miami, Florida
}

(Manuscript received 20 September 2019, in final form 27 January 2020)

\begin{abstract}
A recent study showed observational and numerical evidence for small-scale gravity waves that radiate outward from tropical cyclones. These waves are wrapped into tight spirals by the radial and vertical shears of the tangential wind field. Reexamination of the previously studied tropical cyclone simulations suggests that the dominant source for these waves are convective asymmetries rotating along the eyewall, modulated in intensity by the preferred convection region on the left side of the environmental wind shear vector. A linearized, nonhydrostatic model for perturbations to a balanced vortex is used to study the waves. Forcing the linear model with rotating and pulsing asymmetric heat sources generates radiating gravity waves with multiple vertical and horizontal structures. The pulsation of the rotating heat source generates two types of waves: fast, deep waves with larger radial wavelengths, and slower, secondary waves with shorter radial and vertical wavelengths. The deeper waves produce surface pressure oscillations that have time scales consistent with surface observations, whereas the shorter waves have little surface indication but produce oscillations in vertical velocity with shorter radial wavelengths that are consistent with aircraft observations. Convective forcing that is either not pulsing or not rotating produces gravity waves but they are not as similar to the observed or simulated waves. The effects of varying the intensity of the cyclone, the asymmetry of the forcing, and the static stability of the surrounding atmosphere are explored.
\end{abstract}

\section{Introduction}

As with almost all moist convection in the atmosphere, the deep convection in tropical cyclones (TCs) disturbs the surrounding atmosphere and produces internal gravity waves that radiate outward and upward from their source. Robust, outward-propagating gravity waves were seen in the earliest, three-dimensional numerical simulations of TCs (Anthes 1972; Kurihara and Tuleya 1974; Mathur 1975), and were considered as possible causes for spiral rainbands. However, the large radial wavelengths and fast outward propagation speeds of these simulated waves were not consistent with rainbands as observed in the early weather radars (Wexler 1947; Senn and Hiser 1959). As observations, computer power, and numerical models improved, it became evident that spiral rainbands and gravity waves were distinct processes, and since then spiral rainbands have come to be associated with other convective and dynamical processes (Guinn and Schubert 1993;

Corresponding author: Prof. David S. Nolan, dnolan@rsmas. miami.edu
Montgomery and Kallenbach 1997; Wang 2002; Gall et al. 1998; Nolan 2005; Moon and Nolan 2015a,b).

After the early period of interest in gravity waves as a mechanism for spiral bands, the large majority of research on TCs and gravity waves was concerned with the deeper, longer waves that propagate upward into the stratosphere and mesosphere (e.g., Pfister et al. 1993; Kim and Chun 2005; Kim et al. 2009; Kuester et al. 2008; Chane Ming et al. 2010; Niranjan Kumar et al. 2011; Chane Ming et al. 2019). These generally have wavelengths from tens to hundreds of kilometers. More recently, it has been proposed that measurement of stratospheric gravity wave activity generated by TCs could be used to estimate TC intensity or TC intensity change (Tratt et al. 2018; Hoffmann et al. 2018). There have also been low-level and surface observations of passing gravity waves in the vicinities of TCs (Matsumoto and Okumar 1985; Sato 1993; Niranjan Kumar et al. 2014).

The recent paper by Nolan and Zhang (2017, hereafter NZ17) returned to the investigation of tropospheric gravity waves radiating outwards from TCs. NZ17 found evidence for these waves from in situ measurements of vertical velocity taken by NOAA P-3 aircraft that 

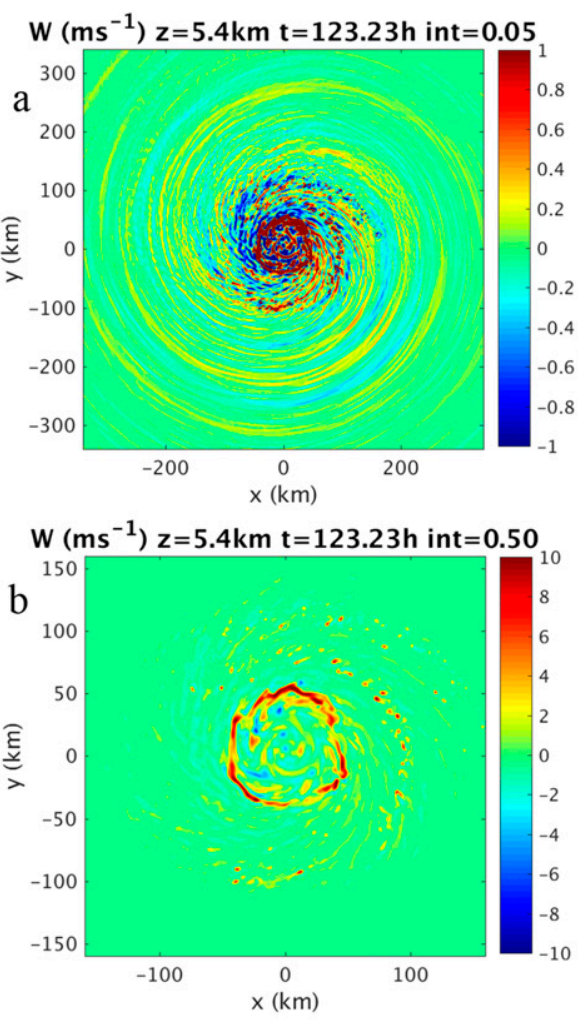

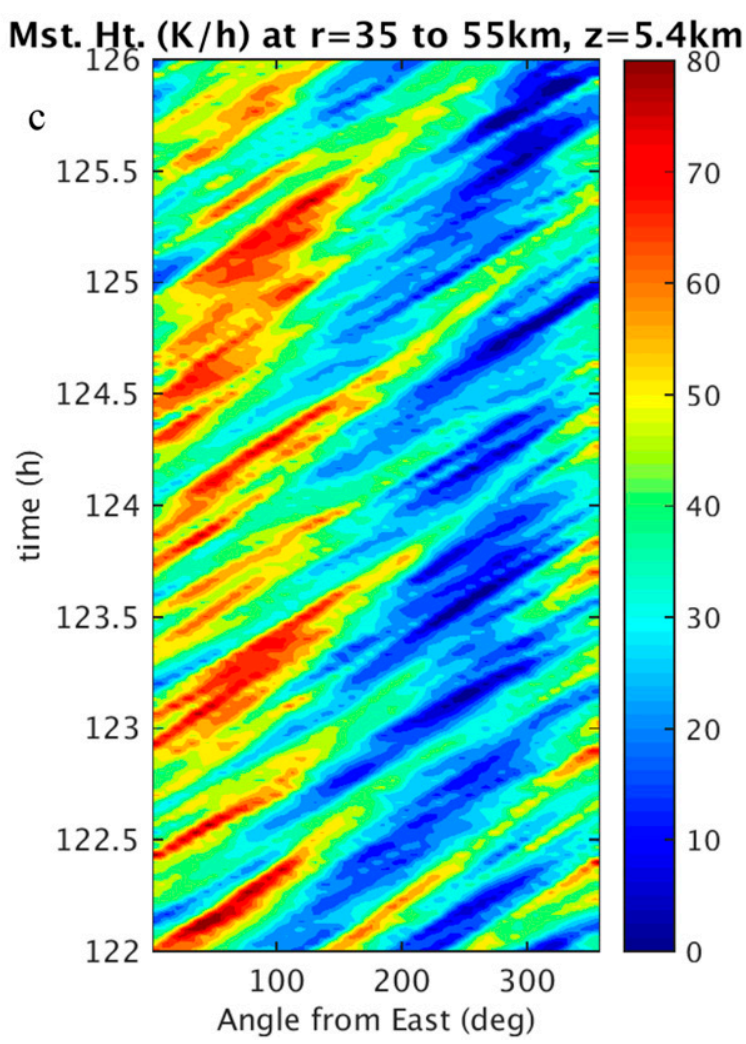

FIG. 1. Vertical velocity $w$ and moist diabatic heating in the idealized hurricane simulation of NZ17: (a) $w$ at $z=$ $5.4 \mathrm{~km}$ on most of the inner nest, with values limited from -1 to $+1 \mathrm{~m} \mathrm{~s}^{-1}$; (b) $w$ at $z=5.4 \mathrm{~km}$ in the eyewall, with color range from -10 to $+10 \mathrm{~m} \mathrm{~s}^{-1}$; (c) azimuth-time Hovmöller diagram of moist heating at $z=5.4 \mathrm{~km}$ and averaged between 35 and $55 \mathrm{~km}$ radius.

penetrate TCs at altitudes such as 1500 and $3000 \mathrm{~m}$, and also in surface observations made from research buoys deployed in the Pacific Ocean. In the latter case, the signals of the waves could be detected in surface pressure and surface winds, even when the TC centers were over $300 \mathrm{~km}$ from the instruments. The aircraft observations showed that these low-level, outward-propagating waves have radial wavelengths of $2-8 \mathrm{~km}$ and phase speeds of $20-30 \mathrm{~m} \mathrm{~s}^{-1}$. Examination of numerical simulations of TCs using $1 \mathrm{~km}$ grid spacing showed outward moving gravity waves with similar properties, although they were underresolved in the simulations, with radial wavelengths about twice as long as seen by aircraft. The simulations show that the waves are wrapped into tight spirals by the radial shear of the tangential flow, and that after their initial generation in the eyewall they were not coupled to moist convection.

Figure 1a shows an example of the vertical velocity $(w)$ field generated in the stronger (category 5) of the two idealized simulations of NZ17. In this plot, the color bar has been set to the narrow range from $w=-1$ to $w=+1 \mathrm{~m} \mathrm{~s}^{-1}$, so that the radiating gravity waves discussed in NZ17 can be seen as the mostly yellow bands that spiral outward from the center. The strong up and down motions in and around the eyewall are saturated (solid red and solid blue). The radiating waves appear to have multiple scales. For example, to the southeast of the eyewall there are three successive bands of mostly upward motion with radial length scales of $20-40 \mathrm{~km}$, but each of these is interspersed with narrow bands of downward motion, creating finer-scale bands with radial scales of $4-10 \mathrm{~km}$.

The purpose of this study is to investigate the basic dynamics of these tropospheric, outward-propagating, spiral gravity waves. The present work is similar to past studies of convectively produced gravity waves that propagate into the stratosphere, such as Fovell et al. (1992), Alexander et al. (1995), and Lane et al. (2001). In particular, Fovell et al. forced a simplified model with momentum sources modeled after cyclic updrafts, and found strong similarities to the waves in their full model. In the stratosphere, waves are modified as they move upward by changes in stratification and by the vertically varying zonal wind. In our case, the waves are modified as they move outward by the strong radial and vertical shears of the tangential wind. Our primary tool will be a 
linear model for time-evolving perturbations to a basicstate flow, where that basic state is a balanced, baroclinic vortex closely modeled after the TC wind field above the boundary layer. The model will be forced by idealized heating functions that are evolving in time as they rotate around the center of the vortex.

Section 2 will describe the inspiration for these heating sources as seen in a full-physics simulation. Section 3 describes the linear model, the basic-state vortex, and the idealized forcing functions. The time-evolving response and associated gravity waves are described in section 4, and sensitivity to atmospheric and forcing parameters are considered in section 5. Section 6 compares the waves in the linear model to the simulations that inspired them. Conclusions are provided in section 7.

\section{The source of gravity waves as seen in a nonlinear model}

NZ17 did not attempt to determine the sources of the outward-propagating waves that were evident in their numerical simulations and in observations. While moist convection in or near the TC eyewall appears to be the source, it is not immediately clear which type of convective structures generate the most prominent waves. Are they caused by the most intense updrafts in the eyewall, with vertical velocities of 10 $20 \mathrm{~m} \mathrm{~s}^{-1}$ and horizontal scales of just a few kilometers (Black et al. 1996; Guimond et al. 2010)? Or are they caused by low-wavenumber asymmetries associated with mesovortices that rotate around the eyewall (Reasor et al. 2000; Nolan and Montgomery 2002; Braun et al. 2006; Hendricks et al. 2012)? Or by the nearly stationary, mostly wavenumber-1 asymmetry caused by enhanced convection in the downshear-left quadrant (Black et al. 2002; Corbosiero and Molinari 2002; Rogers et al. 2003; Reasor et al. 2013)?

To answer this question, we return to one of the TC simulations in NZ17. This simulation used the idealized modeling framework of Nolan (2011), also known as "point downscaling," which allows for specification of vertical profiles of temperature, moisture, and horizontal wind that are initially homogeneous across the model domain, and remain nearly constant through the course of the simulation. In this case, the initial sounding was the Dunion (2011) moist tropical sounding, with the midlevel relative humidity reduced by $20 \%$ so as to limit rainband activity around the storm [this humidity reduction is shown in Fig. 1.6 of Nolan and McGauley (2012)]. The environmental wind profile had $5 \mathrm{~m} \mathrm{~s}^{-1}$ of easterly wind at the surface, with $2.5 \mathrm{~m} \mathrm{~s}^{-1}$ of westerly shear between 850 and $200 \mathrm{hPa}$ [Fig. 1.7 of Nolan and McGauley (2012)]. The sea surface temperature was set to $26.5^{\circ} \mathrm{C}$. The simulation used an outer domain with $27 \mathrm{~km}$ grid spacing, and vortex-following nested grids with 9,3 , and $1 \mathrm{~km}$ grid spacing. The inner nest was $720 \times 720$ grid points. 50 model levels were used between the surface and the model top at $26 \mathrm{~km}$ height. The WRF single-moment 6-class microphysics scheme (WSM6; Hong and Lim 2006) and the Yonsei University (YSU) planetary boundary layer scheme (Hong et al. 2006) were used. The YSU scheme also computes vertical diffusion above the boundary layer based on the wind shear, a fixed length scale, and a stability function that depends on the gradient Richardson number. This stability function keeps vertical mixing close to zero for most of the atmosphere above the boundary layer. A weak, TC-like vortex is introduced at the initial time, and this vortex develops into an intense tropical cyclone with transient surface winds in excess of $70 \mathrm{~m} \mathrm{~s}^{-1}$.

As noted above, Fig. 1a shows an example of the vertical velocity field at $z=5.4 \mathrm{~km}$ during this particular simulation. Given the strong intensity of this simulated TC, it would be expected that the distribution of convection would be highly symmetric about the cyclone center, and at first glance this appears to be the case. However, while the eyewall (seen here as the dark red areas in the figure, indicating updrafts exceeding $1 \mathrm{~m} \mathrm{~s}^{-1}$ ) is generally of the same width in all quadrants, it is slightly more robust on the north (left of shear) side, being slightly broader and with fewer downward motions interspersed. There are convective cells associated with rainbands on both the north and south sides of the storm, but these too are more prevalent on the north side. Therefore, despite the relatively slow motion of the TC (about $4 \mathrm{~m} \mathrm{~s}^{-1}$ to the west) and the very weak environmental wind shear $\left(2.5 \mathrm{~m} \mathrm{~s}^{-1}\right)$, there is a detectable wavenumber- 1 asymmetry in both the eyewall and the surrounding rainbands.

Both small-scale and mesoscale asymmetries in the eyewall are better seen in Fig. 1b, which shows a closeup of the eyewall region, and with the $w$ scale expanded to -10 to $+10 \mathrm{~m} \mathrm{~s}^{-1}$. In this figure we can see the individual updrafts in the eyewall, as well as the broader area of upward motion on the north side, consistent with the left-of-shear preference noted above.

To provide a more robust illustration of the preference for deep convection left of shear, we construct a time-azimuth Hovmöller diagram of moist diabatic heating. The diabatic heating rate at $z=5.4 \mathrm{~km}$ (model level 20) and between radius $r=35$ and $r=55 \mathrm{~km}$ is computed for each azimuth around the storm center from model output at $2 \mathrm{~min}$ intervals. Figure 1c shows that the diabatic heating is greater in the northern quadrant of the eyewall, with strongest heating occurring at angles between $90^{\circ}$ and $120^{\circ}$ counterclockwise 
from east. However, the region of preferred heating is modulated in time by the counterclockwise passage of smaller-scale heating maxima. These convective maxima travel completely around the azimuth approximately every $1.5 \mathrm{~h}$. Alternatively, one could view the heating pattern as having a strong wavenumber-2 (or occasionally wavenumber-3) asymmetry, but that the heating maxima only "turn on" when they pass through the favorable region. Similarly, convection is suppressed on the south side of the eyewall, with mean heating in the $35-55 \mathrm{~km}$ annulus occasionally falling to near zero. This pattern of convection being activated by dynamical asymmetries moving through the downshear-left quadrant was previously identified in studies such as Black et al. (2002), Braun et al. (2006), Braun and Wu (2007), and Reasor et al. (2009).

Analysis of this azimuthal-time Hovmöller diagram and others like it finds that the localized convective regions propagate at speeds of $55-65 \mathrm{~m} \mathrm{~s}^{-1}$. These speeds range from roughly $2 / 3$ to $4 / 5$ of the azimuthal-mean tangential wind speed at the top of the boundary layer, which varies from 80 to $85 \mathrm{~m} \mathrm{~s}^{-1}$ during this stage of the idealized simulation. Therefore, the heat source function used to produce gravity waves in the linear model will be a spatially localized positive heat source that rotates around the center, just inside the radius of maximum winds (RMW), that is also pulsing on and off as it moves through a specified quadrant.

\section{The linear model, basic state, and forcing}

\section{a. $3 D V P A S$}

The model used for this study is known as ThreeDimensional Vortex Perturbation Analysis and Simulation (3DVPAS). This model was first described in Nolan and Montgomery (2002) and Nolan and Grasso (2003), and then later refined in Hodyss and Nolan (2007) and Nolan et al. (2007). 3DVPAS simulates the evolution of linearized perturbations to a stationary basic state, which is a balanced, baroclinic vortex in a frictionless, dry atmosphere, with no surface friction and no boundary layer, and no overturning circulation. The model is derived from anelastic equations in cylindrical coordinates, assuming reference states of temperature $T(r, z)$, pressure $p(r, z)$, and density $\rho(r, z)$, and thus differs slightly from the traditional anelastic equations in that the reference states vary both vertically and horizontally. The far-field temperature field is the Jordan (1958) sounding, with moisture neglected.

The model solves for the motions of each azimuthal wavenumber $n$ separately, with the motions for $n>0$ presumed to be the real parts of complex functions that evolve in time; for example,

$$
w^{\prime}(r, z, \lambda, t)=\operatorname{Re}\left\{w_{n}(r, z, t) e^{i n \lambda}\right\} .
$$

Setting $n=0$ leads to a different equation set with purely real solutions. The motions for each wavenumber can be considered separately, or interpolated to a Cartesian grid and summed to provide the complete solutions.

\section{b. Basic-state vortices and model domain}

The basic-state tangential wind fields $V(r, z)$ are designed to be similar to observed tropical cyclones and are constructed from a combination of theory and arbitrary functions. Specifically, the wind field at the surface is prescribed to follow the modified Rankine vortex profile:

$$
V(r)=\left\{\begin{array}{cc}
V_{m}\left(\frac{r}{\mathrm{RMW}}\right) & r<\mathrm{RMW} \\
V_{m}\left(\frac{\mathrm{RMW}}{r}\right)^{a} & r \geq \mathrm{RMW}
\end{array},\right.
$$

where for most of the simulations the maximum tangential wind $V_{m}=50 \mathrm{~m} \mathrm{~s}^{-1}$, RMW $=50 \mathrm{~km}$, and the decay parameter $a=0.5$, close to the mean value for major hurricanes (Mallen et al. 2005). As the vortex is idealized to be dry and frictionless, there is no secondary circulation, that is, no basic-state radial or vertical flow. Following the algorithm described in the appendix of Moon and Nolan (2010), the wind field is extended into the vertical using the maximum potential intensity theory of Emanuel (1986). This conveniently produces a vertical structure that slopes outward with height, somewhat realistically, without having to resort to additional ad hoc analytical functions such as in Nolan and Montgomery (2002). After the wind field is constructed, an iterative scheme finds the axisymmetric pressure and temperature fields that hold the vortex in hydrostatic and gradient wind balance. These provide the basic-state potential temperature $\theta(r, z)$ and density $\rho(r, z)$.

Figure 2 shows $V(r, z)$ and the deviation of $\theta(r, z)$ from the reference state (the warm core) for the control-case vortex described above. These figures also show the entire model domain, ranging from $r=0$ to $r_{\max }=$ $320 \mathrm{~km}$, and from $z=0$ to $z_{\max }=22 \mathrm{~km}$. Figure 2 also shows the squared Brunt-Väisälä frequency $N^{2}$ for the Jordan sounding (Fig. 2c) and for an idealized sounding with constant values of $N^{2}$ in the troposphere and stratosphere (Fig. 2d). In both cases the tropopause is near $15 \mathrm{~km}$.

The 3DVPAS grid uses 160 equally spaced grid points in the radial direction and 40 equally spaced grid points in the vertical; grid stretching is not used as in some 

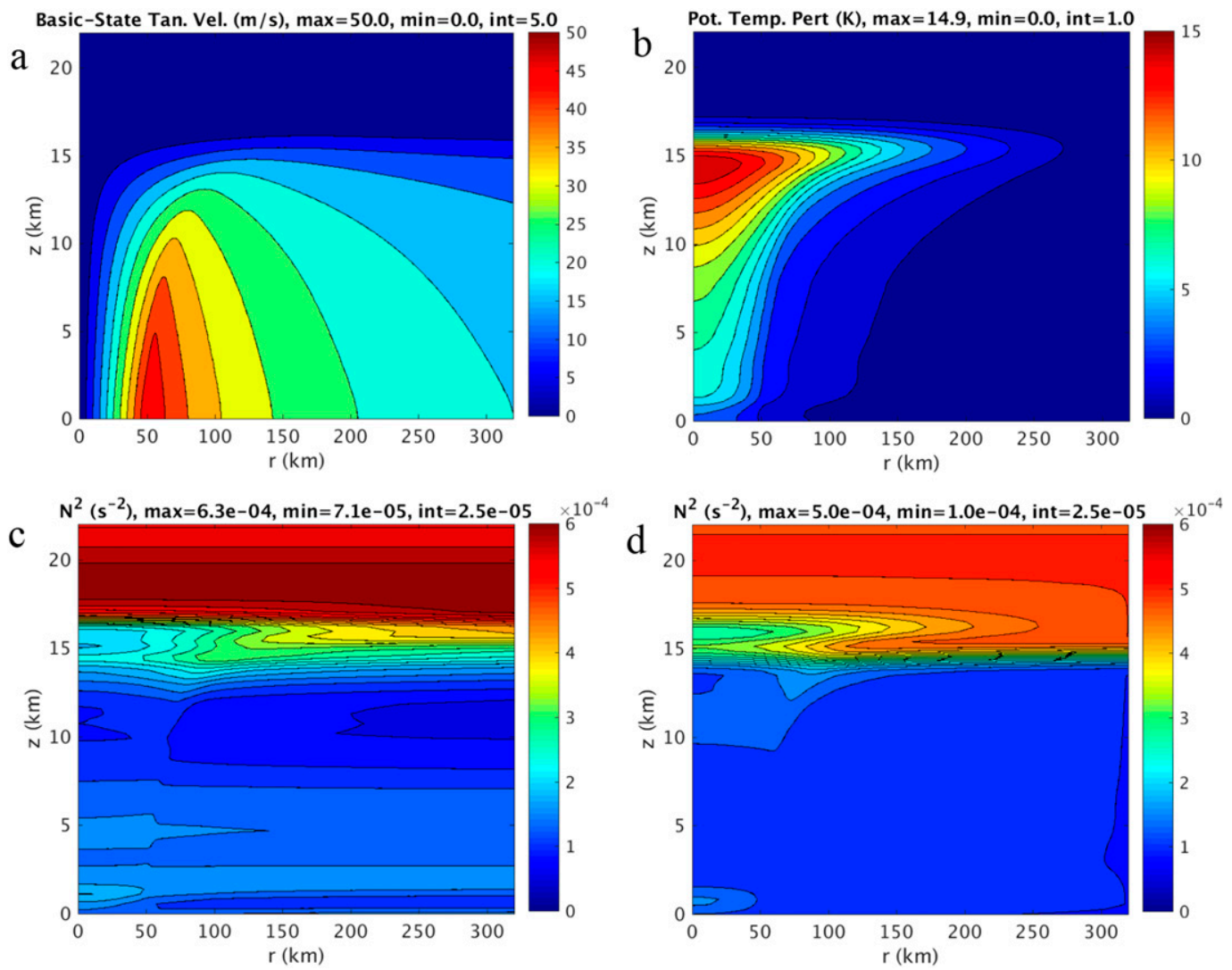

FIG. 2. The basic-state vortex: (a) tangential wind in the radius-height plane; (b) the perturbation potential temperature, relative to the far-field sounding, that holds the vortex in hydrostatic and gradient wind balance; (c) stratification in terms of the Brunt-Väisälä frequency $N$ squared when using the Jordan (1958) sounding; (d) as in (c), but with the far-field sounding using constant $N^{2}=1.0 \times 10^{-4} \mathrm{~s}^{-2}$ in the troposphere.

previous studies. The model applies a constant eddy viscosity to the perturbations with $\nu=20 \mathrm{~m}^{2} \mathrm{~s}^{-1}$.

To mitigate reflection of outward- and upwardpropagating gravity waves, damping zones along the upper and outer boundaries are added to the equations of motion. Using the $\theta$ equation as an example, they have the form

$$
\frac{\partial \theta}{\partial t}=\cdots-\varepsilon(r, z) \theta
$$

with

$$
\begin{aligned}
\varepsilon(r, z)= & \varepsilon_{r} \exp \left[-\left(\frac{r-r_{\max }}{\lambda_{r}}\right)^{2}\right] \\
& +\varepsilon_{z} \exp \left[-\left(\frac{z-z_{\max }}{\lambda_{z}}\right)^{4}\right],
\end{aligned}
$$

where $\varepsilon_{r}=1 / \tau_{r}$ and $\varepsilon_{z}=1 / \tau_{z}$ are inverses of the damping time scales $\tau_{r}$ and $\tau_{z}$. For all calculations in this paper, $\tau_{r}=300 \mathrm{~s}, \tau_{z}=120 \mathrm{~s}, \lambda_{r}=20 \mathrm{~km}$, and $\lambda_{z}=4 \mathrm{~km}$. The same damping is applied to the perturbation velocities.

With the model top at $z=22 \mathrm{~km}$, and the damping region confined to approximately between $z=18$ and $22 \mathrm{~km}$, we should consider whether there is downward reflection of gravity waves or other effects on the results. In appendix A, we show that the results of our simulations are nearly identical if the model top is moved to $z=33 \mathrm{~km}$. The stability of the vortex is demonstrated in appendix B.

\section{c. Time-evolving temperature forcing functions}

Inspired by the rotating and pulsing asymmetries in latent heating as shown above, we use analytical functions to construct heat sources that rotate along the eyewall of the vortex at some fraction of the maximum rotation speed at that radius. In addition, these sources only become "turned on" when they pass through the convectively active downshear-left quadrant. For convenience, we set the center of this region to be due east of the vortex, at azimuth angle $\lambda=0^{\circ}$. 

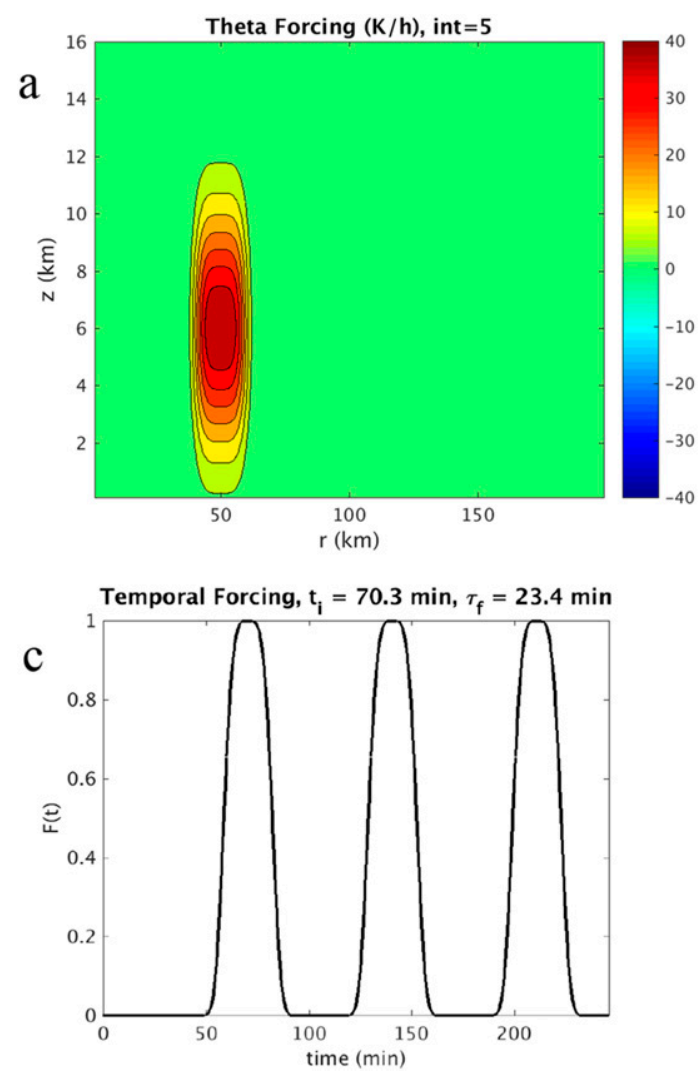

In three dimensions the basic heat source function is

$$
\dot{\theta}(x, y, z)=\frac{\theta_{\text {inj }}}{\tau_{f}} \exp \left[-\left(\frac{d}{\sigma_{r}}\right)^{4}-\left(\frac{z-z_{b}}{\sigma_{z}}\right)^{2}\right],
$$

where $d$ is the distance to the center of the forcing at $x=r_{b}, y=0$,

$$
d=\left[\left(x-r_{b}\right)^{2}+y^{2}\right]^{1 / 2} .
$$

For most of the simulations, the forcing is centered at $r_{b}=50 \mathrm{~km}$ from the vortex center, and at $z_{b}=$ $6 \mathrm{~km}$. The forcing width parameter $\sigma_{r}=10 \mathrm{~km}$ and its depth parameter $\sigma_{z}=4 \mathrm{~km}$. These horizontal and vertical scales are intended to represent the approximate width and depth of the enhanced diabatic heating regions moving around the eyewall as discussed in section 2 . While the vertical scale is about right, the diameter of about $20 \mathrm{~km}$ is larger than what is seen in the model (and in reality), but is the smallest scale that can be consistently well resolved by the linear model. Horizontal and vertical cross sections of this forcing function are shown in Fig. 3. The forcing amplitude is determined by the total change in potential temperature $(\mathrm{K})$ injected by the

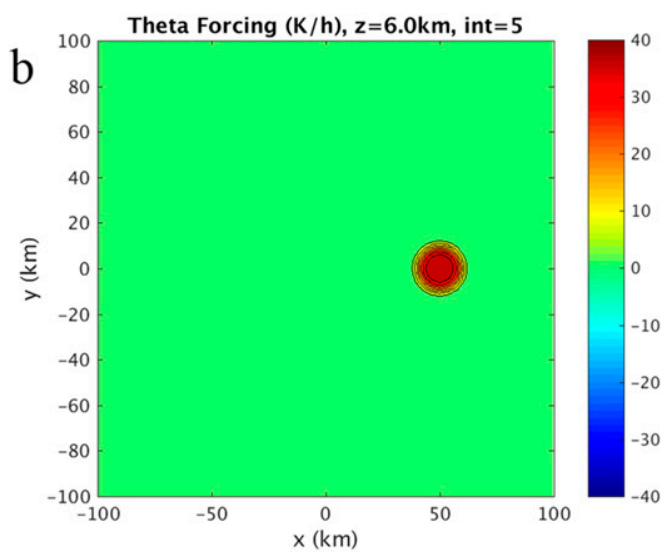

FIG. 3. (a) Vertical and (b) horizontal cross sections of the heat forcing function used in most of the simulations, and (c) an illustration of the temporal forcing function. The maximum heating rate shown occurs only when the timeevolving pulse is at its peak amplitude. heat source, defined by $\theta_{\text {inj }}$, and the time scale of the forcing, defined by $\tau_{f}$.

The time forcing function uses a fourth-power exponential that ramps up quickly but smoothly, is nearly constant for a brief time, and then decreases rapidly back to zero:

$$
F(t)=\exp \left[-\left(\frac{t-t_{i}}{p \tau_{f}}\right)^{4}\right],
$$

where $\tau_{f}$ is the time scale of the forcing period, $t_{i}$ is the time of peak forcing, and choosing $p=0.55163$ means the area under the curve $F(t)$ will be equal to $\tau_{f}$.

The forcing function is also caused to rotate with time around the center of the vortex. In most cases this rotation rate $\Omega_{f}$ is less than the local maximum rotation rate at the specified radius, for example, $\Omega_{f}=0.75 \times \Omega_{\max }$, where $\Omega_{\max }=V\left(r_{b}\right) / r_{b}$. To create this rotation, the forcing function (3.5) is first projected onto each azimuthal wavenumber $n$. Since (3.5) is symmetric about the rotation angle $\lambda=0^{\circ}$, this creates a purely real initial tendency $\dot{\theta}_{n}(r, z)$. Multiplying by the appropriate complex phase rotates the location of the real part of the forcing function to the correct azimuthal angle. Therefore, the timeevolving forcing for each wavenumber is 


$$
\dot{\theta}_{n}(r, z, t)=F(t) \dot{\theta}_{n}(r, z) e^{i n\left(\lambda-\Omega_{f} t\right)} .
$$

Finally, the time of maximum forcing $t_{i}$ and the duration of the forcing $\tau_{f}$ are chosen as follows: we imagine that there are a small number $m$ of equally spaced convective maxima rotating along the eyewall at the angular velocity $\Omega_{f}$, and each of these will turn on as they pass directly east of the center. Therefore, the interval between pulses depends on both $\Omega_{f}$ and $m$; that is,

$$
t_{i}=\frac{2 \pi}{m \Omega_{f}} .
$$

For example, with $m=2$, we imagine there are two convective asymmetries like the one shown in Fig. 3b, but on opposite sides of the center, and each is activated as it passes due east of the center.

We consider both the response to a single pulse and to repeating pulses. For the single-pulse simulations, the pulse occurs only when the first of the two (or more) heat sources approaches $\lambda=0$. For the continuous pulsing simulations, a new pulse occurs near $\lambda=0$ at each additional time interval $t_{i}$ after the first pulse.

For consistency across cases, $\tau_{f}$ is chosen to be some fraction of the pulsing interval $t_{i}$. In most cases we use $\tau_{f}=t_{i} / 3$. For the parameters $V_{m}=50 \mathrm{~m} \mathrm{~s}^{-1}$, $r_{b}=50 \mathrm{~km}, m=2, \Omega_{f}=0.75 \times \Omega_{\max }, t_{i}=2 \pi /\left(m \Omega_{f}\right)=$ $1.17 \mathrm{~h}$, and $\tau_{f}=t_{i} / 3=0.39 \mathrm{~h}$, the time forcing $F(t)$ is shown in Fig. 3c. Note that the first pulse, which should be already occurring at $t=0$, is neglected. All calculations use $\theta_{\text {inj }}=10 \mathrm{~K}$, so that the peak heating rate equals $10 \mathrm{~K} / 0.39 \mathrm{~h}=25.6 \mathrm{~K} \mathrm{~h}^{-1}$, as can be seen in Fig. 3. This heating rate is about $1 / 4$ the peak heating rates in WRF simulation, as can be inferred from Fig. 1c (but noting that the data there are averaged across a $20 \mathrm{~km}$ radial distance). Since the model is linear, the equivalent response to greater heating can easily be computed.

\section{Gravity waves from rotating, transient heat sources}

\section{a. Single-pulse forcing and associated gravity wave radiation}

The first case uses the standard parameters described above to produce a single pulse of heating that does not repeat. The response is computed separately for each wavenumber from $n=0$ to $n=5$. The solutions are evolved from $t=0$ to $t=12 \mathrm{~h}$ with a $60 \mathrm{~s}$ time step, using fourth-order Runge-Kutta integration in time of the linear dynamical system.
Figure 4 shows the vertical velocity $w$ at $z=6.3 \mathrm{~km}$ for selected times. The first is $t=t_{i}$, and the heat source is evidently driving a strong upward motion with maximum $w$ of $0.95 \mathrm{~m} \mathrm{~s}^{-1}$ due east of the center. Downward motions are evident immediately upstream and downstream of the upward velocity maximum, with alternating signals of weakening positive and negative vertical motion extending around to the other side of the eyewall. These are not remote responses to the forcing, but rather they are residuals that appear due to the cutoff of the wavenumber summation at $n=5$. Increasing the maximum wavenumber causes these residuals to become smaller and weaker with each successive wavenumber (not shown). There is also a weaker downward motion immediately to the northeast of the updraft. This motion is physical and will become the leading edge of the dominant wave packet.

Only 20 min later at $t=1.5 \mathrm{~h}$ the pulse has expanded into several distinct wave peaks propagating outward, but that also have been advected cyclonically. The leading wave of downward motion has already begun to take a spiral shape. At the same time, the peak upward motion (at this altitude) has already decreased to $0.15 \mathrm{~m} \mathrm{~s}^{-1}$. By $t=2.0 \mathrm{~h}$, the leading edge wave has been advected to northwest of the TC center, during which the peak of the downward motion at the center of the wave has moved radially outward a distance of approximately $70 \mathrm{~km}$. Over the next few hours, the leading wave continues to propagate away rapidly, while waves with shorter radial lengths move more slowly and are left behind as their amplitudes continue to decrease. By $t=4 \mathrm{~h}$, the initial packet is gone, while slow-moving, short radial-scale waves remain with amplitudes decreasing to $0.012 \mathrm{~m} \mathrm{~s}^{-1}$.

A complementary view of this dispersive wave train can be seen by projecting the solutions onto radiusheight sections defined by a specified azimuth. As the majority of the wave energy also is also advected around the vortex as it propagates outward, here we choose the azimuthal angles $\lambda=135^{\circ}, 180^{\circ}, 225^{\circ}$, and $270^{\circ}$ from due east, that is, defining rays going northwest, west, southwest, and south of the TC center, at $t=2.0,2.5,3.0$, and $4.0 \mathrm{~h}$, that is, matching Figs. $4 \mathrm{c}-\mathrm{f}$. These vertical cross sections are shown in Fig. 5. In Fig. 5a, the large radial wavelength and vertical coherence of the leading gravity wave is evident, while behind it there appears to be a jumbled mix of interfering waves. Making similar plots for each wavenumber alone (not shown) reveals that most of the leading wave comes from $n=0,1$, and 2, while most of the high-frequency waves behind it comes from $n=3,4$, and 5 . At $t=2.5 \mathrm{~h}$, the leading wave is exiting the domain, while the secondary wave packet appears to have been filtered into a cleaner set of shorter 

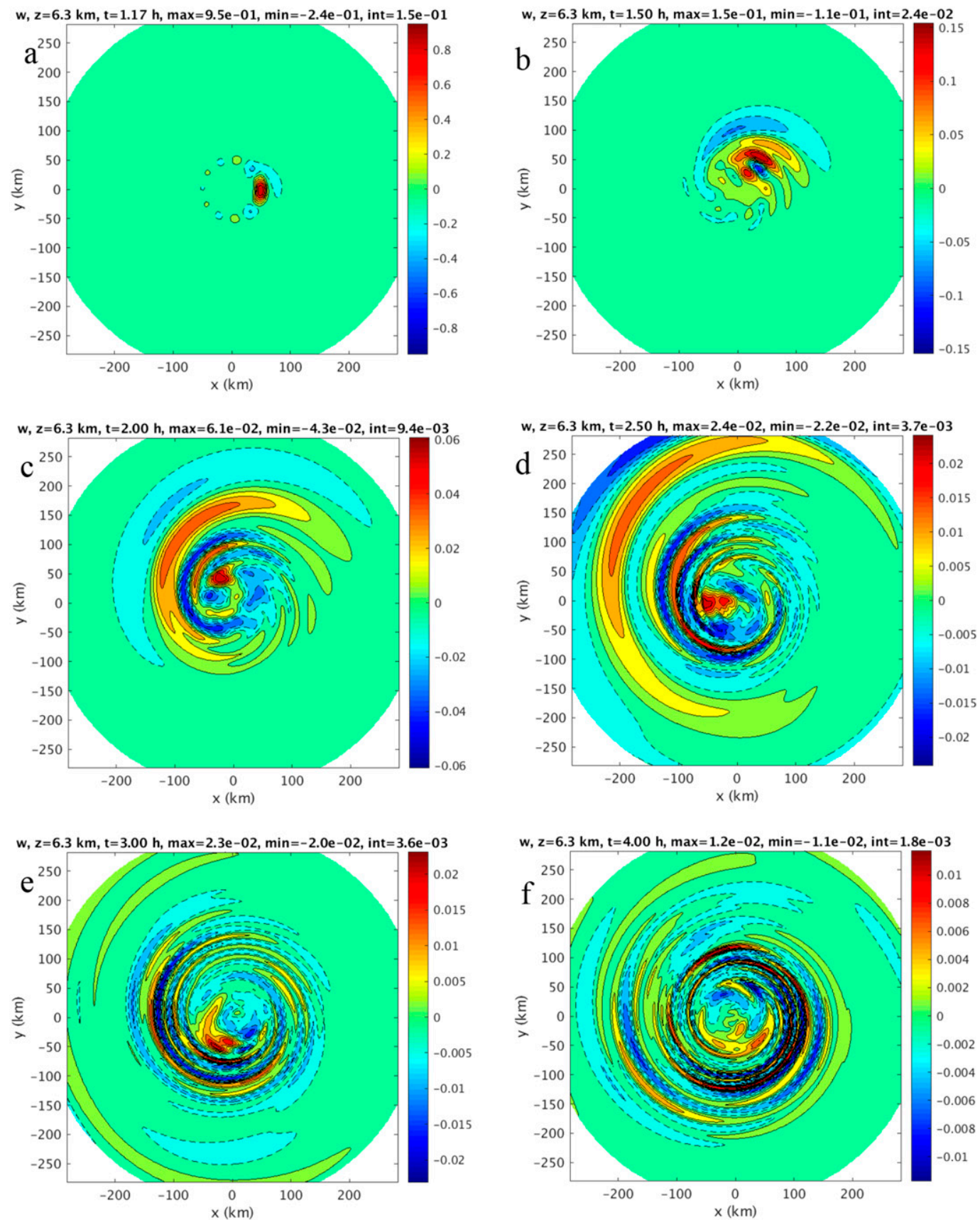

FIG. 4. Vertical velocity $\left(\mathrm{m} \mathrm{s}^{-1}\right)$ in response to the control case with standard single-pulse forcing, at $z=6.3 \mathrm{~km}$, at times $t=$ (a) 1.17, (b) 1.50, (c) 2.00, (d) 2.50, (e) 3.00, and (f) $4.00 \mathrm{~h}$. Note that contours and colors are scaled to the maximum value of $w$ on each plot.

waves with phase lines tilting outward, indicating outward and downward phase speeds and outward and upward group velocities. These secondary waves are most prominent and most coherent at about 11 to $12 \mathrm{~km}$ altitude, which is well above the maximum of the forcing function (at $z=6 \mathrm{~km})$, but below the tropopause around $z=14.5 \mathrm{~km}$ (see Fig. 2c). Over the next $1.5 \mathrm{~h}$, these waves move outward very slowly and quickly decay in amplitude.

This pattern of a series of gravity waves with decreasing vertical and horizontal wavelengths produced by a transient heat source is well known. Nicholls et al. (1991) and Mapes (1993) found that transient heat sources in a Boussinesq, resting atmosphere produce a 

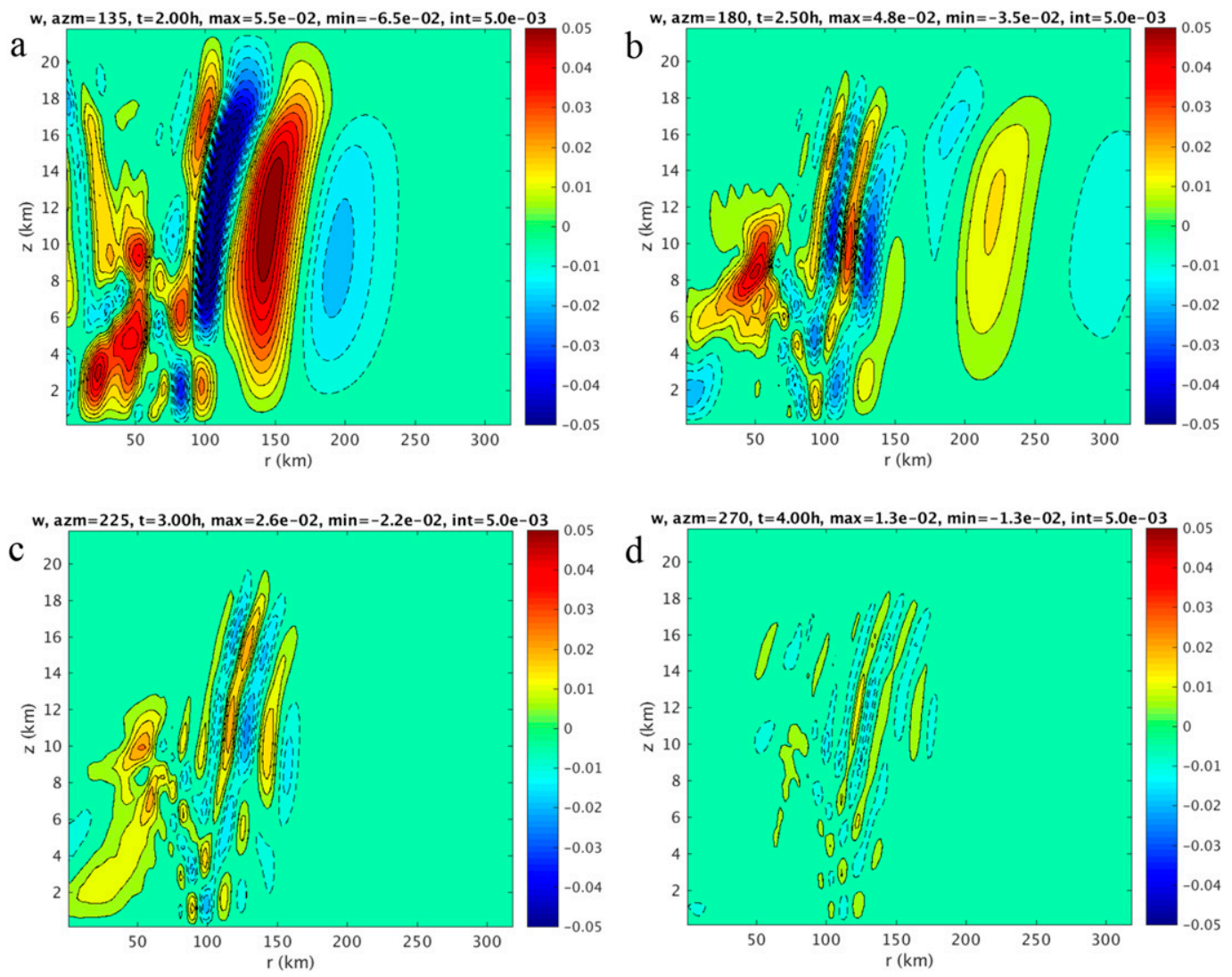

FIG. 5. Radius-height cross sections of $w$ along specified azimuths for the control case, single pulse forcing: (a) $w$ along $\lambda=135^{\circ}$ at $t=2.0 \mathrm{~h}$; (b) $w$ along $\lambda=180^{\circ}$ at $t=2.5 \mathrm{~h}$; (c) $w$ along $\lambda=225^{\circ}$ at $t=2.0 \mathrm{~h}$; (d) $w$ along $\lambda=270^{\circ}$ at $t=2.0 \mathrm{~h}$. Contour intervals and color ranges are fixed in these plots.

primary, deep wave moving a way from the source, followed by a secondary, baroclinic wave. Using a more realistic model, Alexander and Holton (2004) found that a broader spectrum of waves will be excited by a transient heat source, but also with the pattern of the decreasing vertical wavelengths.

Looking across the previous two figures, we see that the amplitudes of the waves in $w$ vary from as much as $0.06 \mathrm{~m} \mathrm{~s}^{-1}$ at upper levels in the leading wave, to values such as $0.02 \mathrm{~m} \mathrm{~s}^{-1}$ in the secondary waves, and then decreasing with time. In a linear model, the response is exactly proportional to the forcing, and we noted above that the peak heating rate of the forcing $\left(25.6 \mathrm{~K} \mathrm{~h}^{-1}\right)$ is about $1 / 4$ of the peak heating rates from the WRF simulation. With equivalent forcing, 3DVPAS would produce $w$ oscillations reaching about $0.25 \mathrm{~m} \mathrm{~s}^{-1}$. The peak amplitudes of the $w$ waves are around $0.5 \mathrm{~m} \mathrm{~s}^{-1}$ in the simulation, and the differences are at least partly due to the lower resolution and greater diffusion in 3DVPAS. Even stronger oscillations, as large as $1.0 \mathrm{~m} \mathrm{~s}^{-1}$, and were seen in aircraft observations (NZ17). From radar observations, Guimond et al. (2011) diagnosed local heating rates over $200 \mathrm{~K} \mathrm{~h}^{-1}$ in the case of Hurricane Guillermo (1997). Therefore, the WRF simulations may also underestimate the intensity of the convective asymmetries.

NZ17 also found signals of gravity waves in TCs from measurements of surface pressure. Although pressure is eliminated from the 3DVPAS equations, it can be computed from the state variables and their tendencies (see appendix A of Nolan and Montgomery 2002). We use pressure $p$ at the lowest model half level $(z=0.3 \mathrm{~km})$ as a proxy for the surface pressure. These $p$ perturbations are shown in Fig. 6, at the same times as the $w$ perturbations shown in Fig. 4. The net positive heating of the forcing function generates a negative pressure perturbation at the surface, and shortly afterward there is a clear surface signal from the leading gravity wave as it moves outward from the center. However, the secondary wave packet has minimal indication at the surface due to its shorter radial wavelengths and higher vertical wavenumbers (as shown in Fig. 5). Instead, the surface pressure anomaly that is still visible at later times such as $t=3 \mathrm{~h}$ and $t=4 \mathrm{~h}$ is the residual 

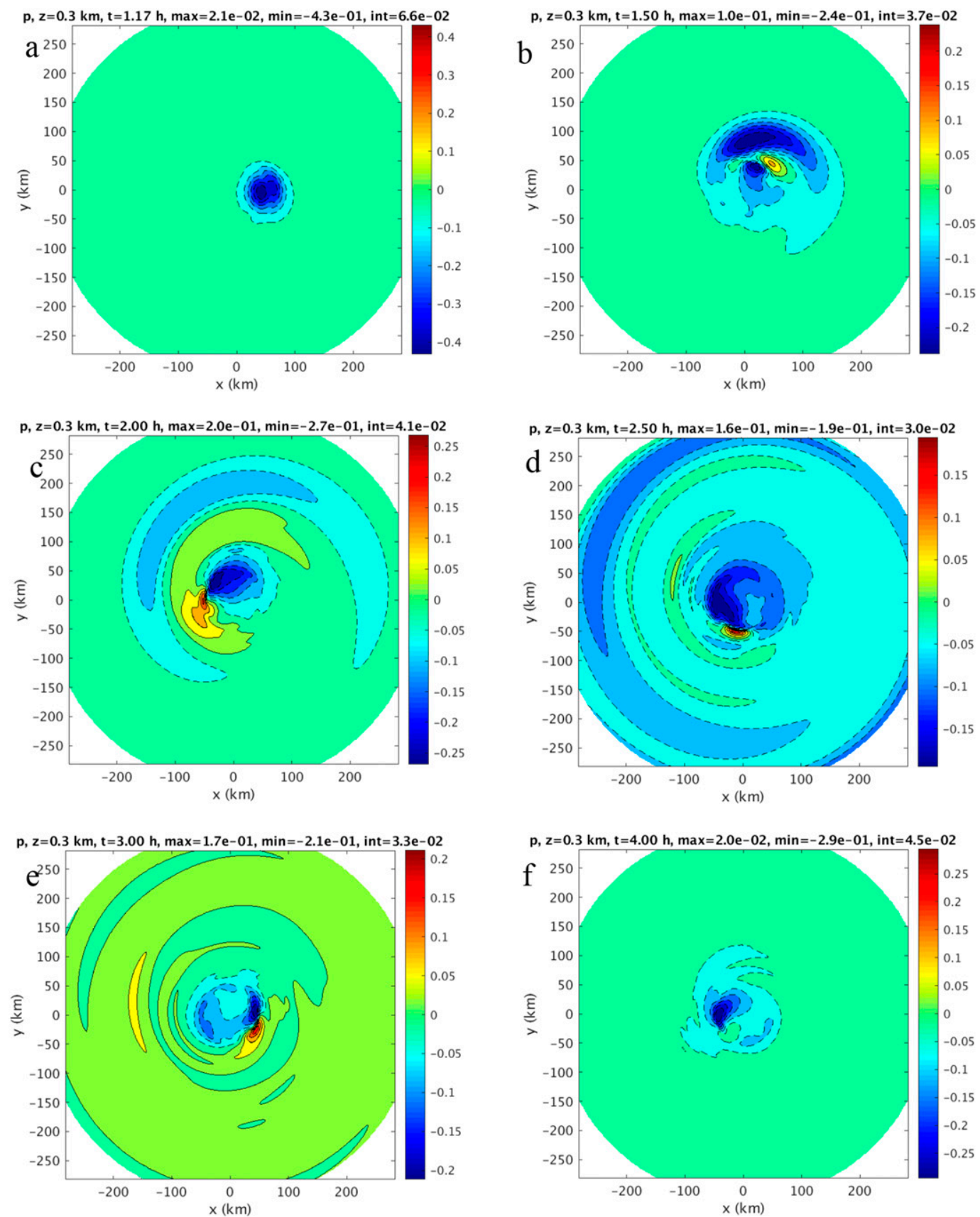

FIG. 6. As in Fig. 4, but for near-surface pressure perturbations (Pa).

negative anomaly associated with the dynamical adjustment to the net positive heating of the forcing function. This is the final stage of the asymmetric intensification process studied in Nolan and Grasso (2003) and Nolan et al. (2007). The surface pressure reduction due to the symmetric adjustment can be eliminated by removing $n=0$ from the summed solutions, as shown in Fig. 7a; but surprisingly, the surface pressure anomalies associated with the radiating waves are still dwarfed by larger-amplitude, long-lived asymmetries excited in the inner core of the vortex by the initial disturbance. Surface perturbations associated with the secondary waves can be better revealed by simply masking out the solution inside $r=100 \mathrm{~km}$, as shown in Fig. 7b.

A question of theoretical interest is how well these waves are captured by essentially hydrostatic dynamics, or if their behavior is strongly influenced by nonhydrostatic 

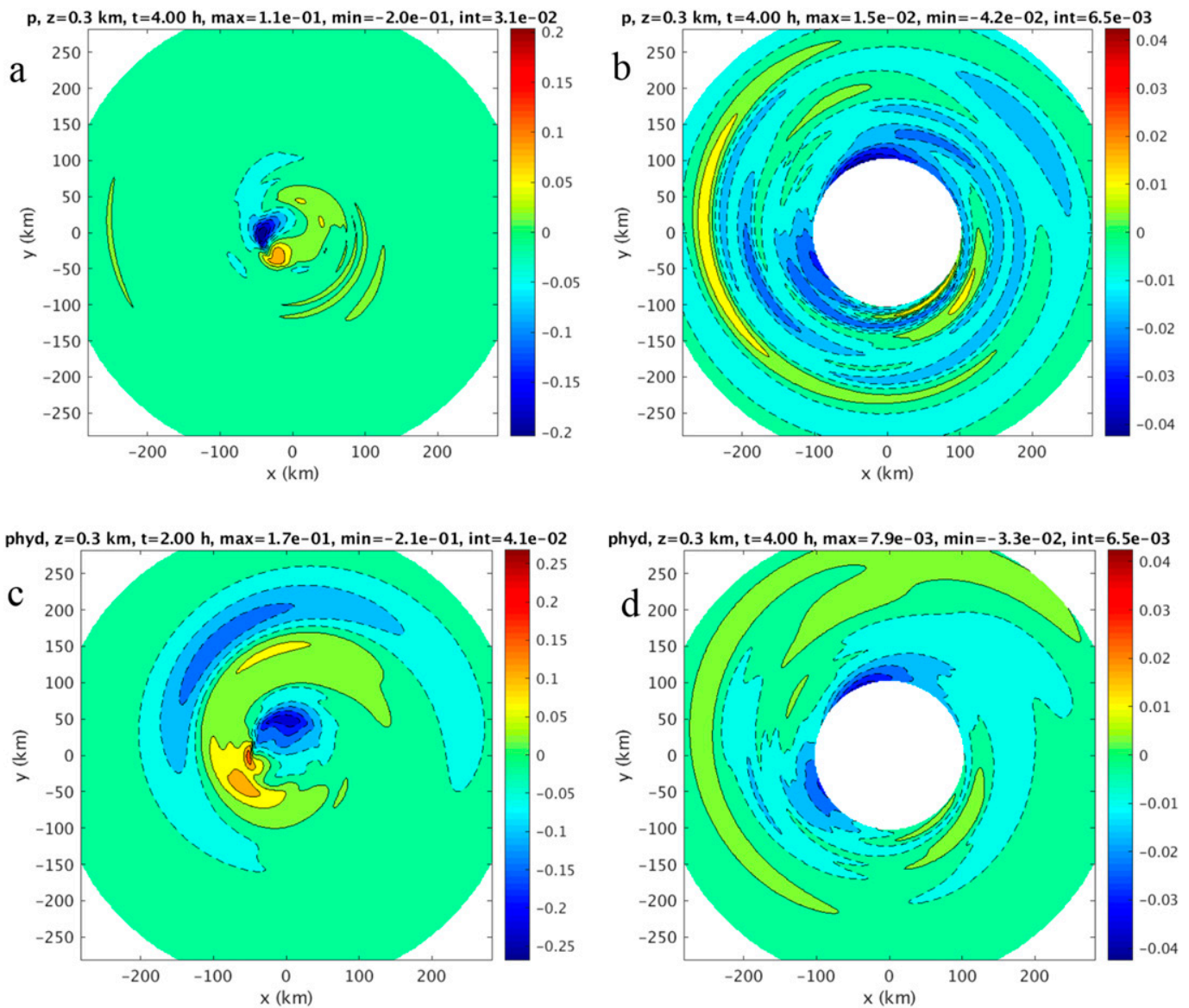

FIG. 7. Selected modifications to the near-surface pressure perturbations: (a) at $t=4.0 \mathrm{~h}$ with the $n=0$ contribution removed; (b) at $t=4.0 \mathrm{~h}$ with $n=0$ included but the data inside $100 \mathrm{~km}$ masked; (c) the hydrostatic $p$ at $t=2.0 \mathrm{~h} ;(\mathrm{d})$ the hydrostatic $p$ at $t=4.0 \mathrm{~h}$ with the data inside $100 \mathrm{~km}$ masked. Units are $\mathrm{Pa}$.

effects. Many textbooks (e.g., Vallis 2006; Sutherland 2010) use the dispersion relation for simple plane waves in a resting atmosphere with constant stratification,

$$
\omega^{2}=\frac{N^{2} k^{2}}{\left(k^{2}+m^{2}\right)}
$$

(where $\omega$ is the frequency and $k$ and $m$ are the horizontal and vertical wavenumbers) and its equivalent expression derived from hydrostatic equations,

$$
\omega^{2}=\frac{N^{2} k^{2}}{m^{2}}
$$

to demonstrate that gravity waves behave "hydrostatically" when $k^{2}$ is small compared to $m^{2}$, that is, when the horizontal wavelengths are considerably longer than the vertical. The horizontal wavelengths can be estimated from the radial widths of positive and negative perturbations along a specified ray emanating from the center, in the form of radius-time Hovmöller diagrams. Figures $8 \mathrm{a}$ and $8 \mathrm{~b}$ show $w$ at $z=11.8 \mathrm{~km}$ and $p$ at $z=0.3 \mathrm{~km}$ along azimuth $\lambda=135^{\circ}$. From these figures we can estimate a horizontal wavelength $L_{x}=120 \mathrm{~km}$ (twice the radial width of the leading negative $p$ anomaly), while from Fig. 5 we can estimate $L_{z}=40 \mathrm{~km}$ (twice the height of the leading positive $w$ anomaly), and therefore $k^{2} \sim 11 \%$ of $\mathrm{m}^{2}$. This suggests the leading wave is approximately hydrostatic.

We can also compare the extent to which the pressure fields caused by the waves are similar to their associated hydrostatic pressure perturbations $p_{\text {hyd }}$; this term can be computed by downward integration of the temperature perturbations. Figure $7 \mathrm{c}$ shows nearsurface $p_{\text {hyd }}$ at $t=2.0 \mathrm{~h}$, which should be compared to $p$ in Fig. 6c. At this time, the fields are quite similar, indicating that the leading wave and the inner-core adjustment process are well captured by hydrostatic dynamics. However, at $t=4.0 \mathrm{~h}, p_{\text {hyd }}$ outside the inner core, as shown in Fig. 7d, is quite different from $p$ in Fig. 7b, showing that the shorter-wavelength, slowermoving $w$ waves are less hydrostatic. 

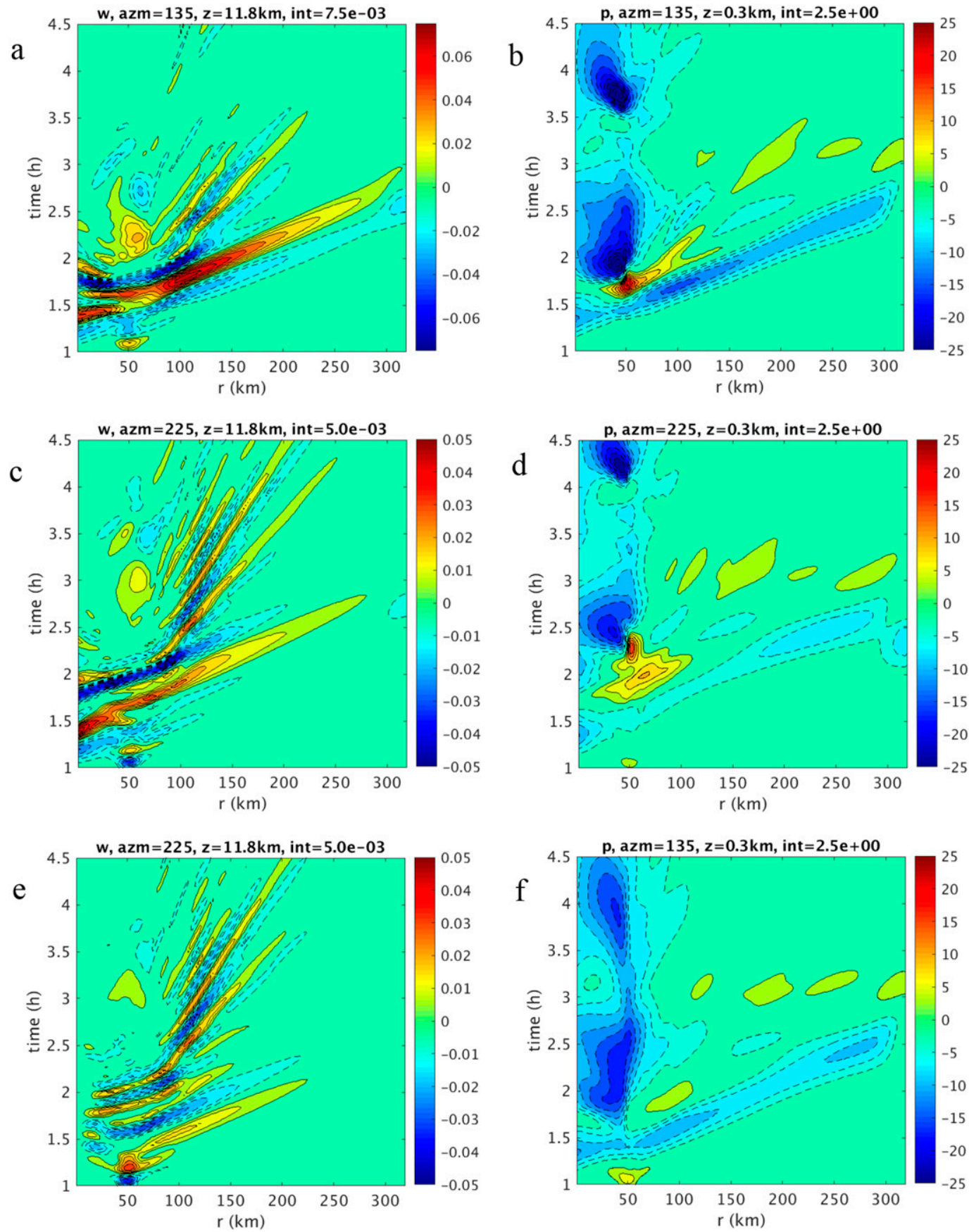

FIG. 8. Radius-time Hovmöller diagrams of $w$ at $z=11.8 \mathrm{~km}$ and $p$ at $0.3 \mathrm{~km}$ : (a) $w$ from all wavenumbers along $\lambda=135^{\circ}$; (b) $p$ from all wavenumbers along $\lambda=135^{\circ}$; (c) $w$ from all wavenumbers along $\lambda=225^{\circ}$; (d) $p$ from all wavenumbers along $\lambda=225^{\circ}$; (e) $w$ from $n=2$ to 5 along $\lambda=225^{\circ}$; (f) $p$ from $n=0$ and 1 along $\lambda=135^{\circ}$.

Figures $8 \mathrm{a}$ and $8 \mathrm{~b}$ also show the robust and steady propagation of the leading wave. From these figures we can estimate the outward phase speed of the leadingedge negative pressure anomaly to be approximately $69 \mathrm{~m} \mathrm{~s}^{-1}$. If we use $N=0.012 \mathrm{~s}^{-1}$, and the same $L_{x}$ and
$L_{z}$ as above, we can estimate from the horizontal phase speed:

$$
c_{x}=\frac{\omega}{k}=\frac{N}{\left(k^{2}+m^{2}\right)^{1 / 2}} \approx 72 \mathrm{~m} \mathrm{~s}^{-1},
$$


which comes very close to what is seen in Fig. 8b. Remarkably there seems to be little modification of the wave speed due to cylindrical geometry, the vertically varying stratification, or advection by the basic-state flow. This is emblematic of the "high Froude number" approximation used in many studies of gravity waves in TC-like vortices (e.g., Schecter and Montgomery 2004; Schecter 2008), which simply says that the waves move too fast to be significantly affected by the vortex. A secondary, positive pressure wave can be seen following behind the leading wave, with phase speed of approximately $25 \mathrm{~m} \mathrm{~s}^{-1}$.

Figures $8 \mathrm{c}$ and $8 \mathrm{~d}$ show $w$ and $p$ radiating outward along $\lambda=225^{\circ}$. The $w$ component of the leading edge wave is present, although it appears later than the waves in Fig. $8 \mathrm{a}$ because $\lambda=225^{\circ}$ is rotated downstream from $\lambda=135^{\circ}$. After that, a series of shorter, slower waves radiate out of the TC core, with speeds ranging from 17 to $10 \mathrm{~m} \mathrm{~s}^{-1}$. The speeds of each successive wave are reduced by their decreasing radial and vertical wavelengths.

To determine how much the symmetric and asymmetric responses contribute to the radiating waves, we generate similar Hovmöller diagrams summed over a limited set of wavenumbers. For example, Fig. $8 \mathrm{f}$ shows the surface pressure along $\lambda=135^{\circ}$ using only $n=0$ and $n=1$. This leading edge wave is similar in structure and amplitude to that which results from the sum over all wavenumbers (Fig. 8b). In contrast, Fig. 8e shows $w$ along $\lambda=225^{\circ}$ using $n=2$ to 5 . Much of the leading wave is lost, whereas the secondary waves are still well represented.

\section{b. Comparison to the no-vortex response}

The results of the previous section depict a complex response to the forcing from a single pulse of net positive heating that is simultaneously rotating along the RMW of a balanced, tropical cyclone-like vortex. The pulse initially generates a vertically deep gravity wave that radiates outward with a phase speed of about $70 \mathrm{~m} \mathrm{~s}^{-1}$. This leading wave is fairly symmetric, but the displacement of the heat source from the vortex center and a small degree of horizontal shearing from the primary circulation cause this wave to become somewhat asymmetric with significant contributions from $n=1$ and, to a lesser extent, the higher wavenumbers. Behind this, a wave packet with shorter radial and vertical wavelengths appears, with each wave moving more slowly as they are sheared into tighter spirals over time. These secondary waves are evident in the $w$ field but have minimal indication at the surface.

How much of this gravity wave response is due to the complexity of the basic state (the balanced vortex), and how much of it is simply emblematic of the response to any isolated pulse of heating in the atmosphere? As it turns out, much of the behavior is consistent with an isolated pulse in a resting environment. To show this, we compute the response to a heat source identical to that used in the previous section, but centered at $r=0$, and with the basic-state vortex eliminated. This produces a purely symmetric response with $n=0$ motions only. The $w$ fields at $t=1.0,2.0$, and $3.5 \mathrm{~h}$ are shown in Fig. 9, along with a radius-time Hovmöller diagram similar to those shown above, except that the data inside $r=80 \mathrm{~km}$ have been masked out so that the radiating waves can be seen more clearly. There are strong similarities between the radius-height structures of these symmetric waves and the radial cross sections shown in Fig. 5. The Hovmöller is also similar to those shown in Fig. 8, although the purely symmetric waves in Fig. 9 show less distinction in structure and propagation speed between the leading edge wave and the secondary waves. This may be because the slower-moving secondary waves are symmetric and are not distorted by shear of the tangential flow.

\section{c. The response to steady pulsing}

If the forcing function pulses repeatedly, a repeating field of gravity waves radiates outward from the center. The deep, primary waves and the shallow, secondary waves become mixed together, as the fast waves from the most recent pulse will overtake the secondary waves from the previous pulses. The repeating pulses represent the heat released when each of the two convective maxima pass through the favorable region. For the control case this occurs every $t_{i}=1.17 \mathrm{~h}$ (as shown in Fig. 3c). After $6 \mathrm{~h}$ the wave field repeats steadily with the same period as the pulsing.

Figure 10 shows the steady, radiating gravity wave field generated by the pulsing heat source. The plots on the left show snapshots of $w$ at $z=11.8 \mathrm{~km}$ and $z=$ $3.0 \mathrm{~km}$, and $p$ at $0.3 \mathrm{~km}$. The altitude of $11.8 \mathrm{~km}$ is chosen to be representative of the waves that have been observed in TC outflows (NZ17; Doyle et al. 2017), while $3.0 \mathrm{~km}$ is the altitude at which the NOAA P3 aircraft most frequently make observations. Due to the relatively low resolution of our linear model, these waves have larger radial wavelengths than observed (e.g., 4$6 \mathrm{~km}$ at flight level in NZ17). At both altitudes the most recently generated "fast wave" can be seen to the northwest of the center, with old slow waves ahead of it and new slow waves behind it; its amplitude is greater at $11.8 \mathrm{~km}$. The surface signal of the deep wave can also be seen in the surface pressure field.

Figures 10d-f show Hovmöller diagrams along $\lambda=180^{\circ}$ at each altitude. The fast wave is most prominent in the surface pressure, while the slow waves are more 

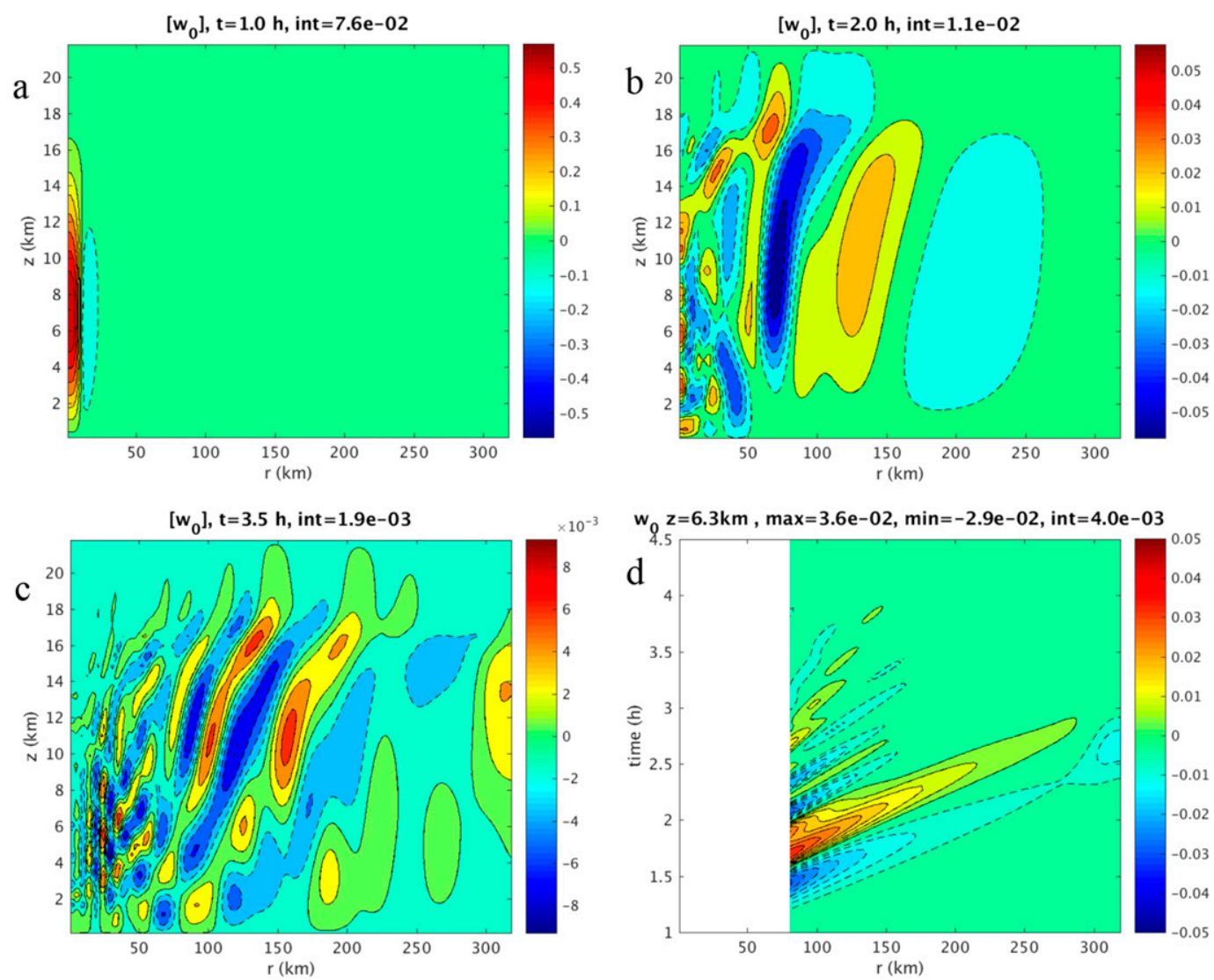

FIG. 9. Symmetric $w$ response produced by a single isolated pulse at $r=0$ : radius-height cross sections of $w$ at $t=$ (a) 1.0, (b) 2.0, and (c) $3.5 \mathrm{~h}$; and (d) radius-time Hovmöller diagram of $w$ at $z=6.3 \mathrm{~km}$.

prominent at $z=3.0 \mathrm{~km}$, and at $11.8 \mathrm{~km}$ we can see a mix of both.

Taking data from along constant radius on the Hovmöller diagrams is equivalent to producing a time series at a fixed point relative to the vortex. This is useful because it allows for a direct comparison to the only quantitative observations we have of these waves, which are the fixed-point time series from surface instruments and the flightlevel data from NOAA aircraft (which were moving, but we can account for their motion). We consider these time series at six locations: at radii of 120 and $240 \mathrm{~km}$, and at azimuths of $0^{\circ}, 135^{\circ}$, and $225^{\circ}$. Figure 11 shows time series of surface pressure and $w$ at $3.0 \mathrm{~km}$ from $t=6$ to $12 \mathrm{~h}$ at each point.

The results show similarities and differences across all the locations. First, all the signals show a dominant periodicity at the pulsation period of $1.17 \mathrm{~h}$. Most, but not all, show secondary signals at half this period, and some show higher frequencies as well. In general, the $w$ series show more energy at higher frequencies as compared to the series of $p$, which except in one case (Fig. 11a) only show one additional peak in addition to the dominant period.

Given what we have seen in the previous sections, it is not difficult to offer a qualitative interpretation of these various signals. The asymmetric eyewall pulsation produces a primary, deep, and fast wave that has a strong reflection in surface pressure. This provides the primary signal seen in the surface pressure. In addition to the fast waves, there are waves with shorter radial wavelengths but that also move outward more slowly. These additional waves are more evident in the $w$ series. As the waves are advected tangentially while they radiate outward, the signals can be quite different at different radii along the same azimuth. This is illustrated in Figs. $11 \mathrm{c}$ and $11 \mathrm{~d}$, where high-frequency waves that are present at $r=120 \mathrm{~km}$ are not visible at $r=240 \mathrm{~km}$. The reverse relationship is seen at $\lambda=0^{\circ}$ (Figs. 11e,f), where higher-frequency waves are present at the large radii. These are shorter waves that have been advected entirely around the vortex.

Despite their complexity, and point-to-point variability, these time series are qualitatively consistent with the analyses of in situ observations shown in NZ17. At flight level, $w$ was dominated by short waves with radial wavelengths of $4-8 \mathrm{~km}$ moving outward at phase speeds of 20-25 m s${ }^{-1}$, with local periods of $4-5 \mathrm{~min}$. None of the signals shown in Fig. 11 have periods this short, but the 

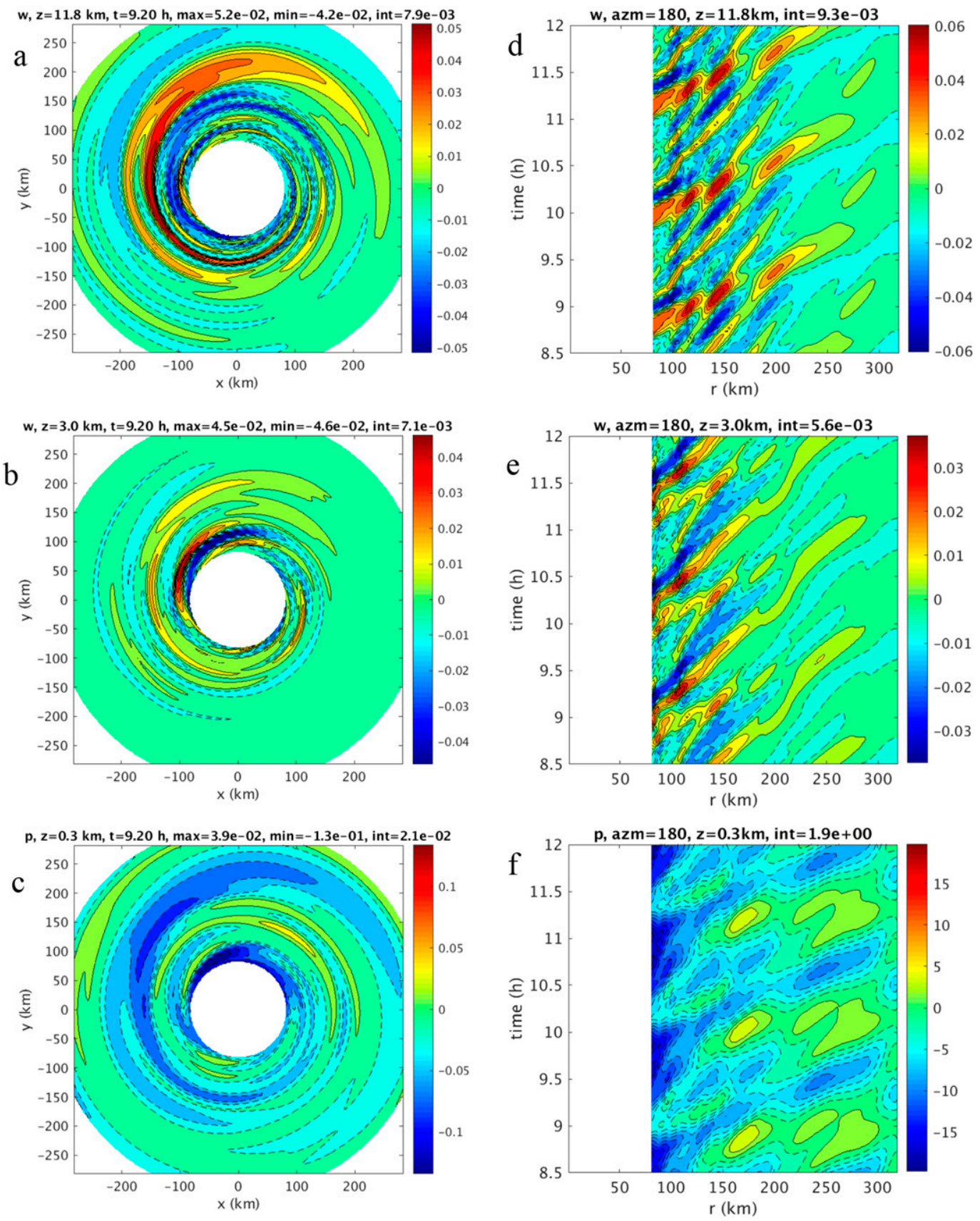

FIG. 10. Results with forcing that repeats: (a) $w$ at $z=11.8 \mathrm{~km}$, (b) $w$ at $z=3.0 \mathrm{~km}$, (c) $p$ at $z=0.3 \mathrm{~km}$ at $t=9.20 \mathrm{~h}$; and radius-time Hovmöller diagrams of (d) $w$ along azimuthal angle $\lambda=180^{\circ}$ at $z=11.8 \mathrm{~km}$, (e) $w$ at $z=3.0 \mathrm{~km}$, and (f) $p$ at $z=0.3 \mathrm{~km}$. Color ranges and contour intervals are selected arbitrarily for each case and are indicated on each plot.

radial wavelengths in our low-resolution model are larger than observed. At the surface, the pressure is dominated by a low frequency with period of about $1 \mathrm{~h}$, with some power in one additional shorter frequency. This is very similar to what is seen in NZ17 (see their Fig. 5), where some of the surface pressure power spectra show two peaks, one around $3000 \mathrm{~s}$ and another around $1000 \mathrm{~s}$.

\section{Sensitivity to vortex and environmental parameters}

\section{a. Vortex intensity}

NZ17 suggested it might be possible to infer TC intensity from analysis of radiated gravity waves. How do the results above vary with TC intensity? 

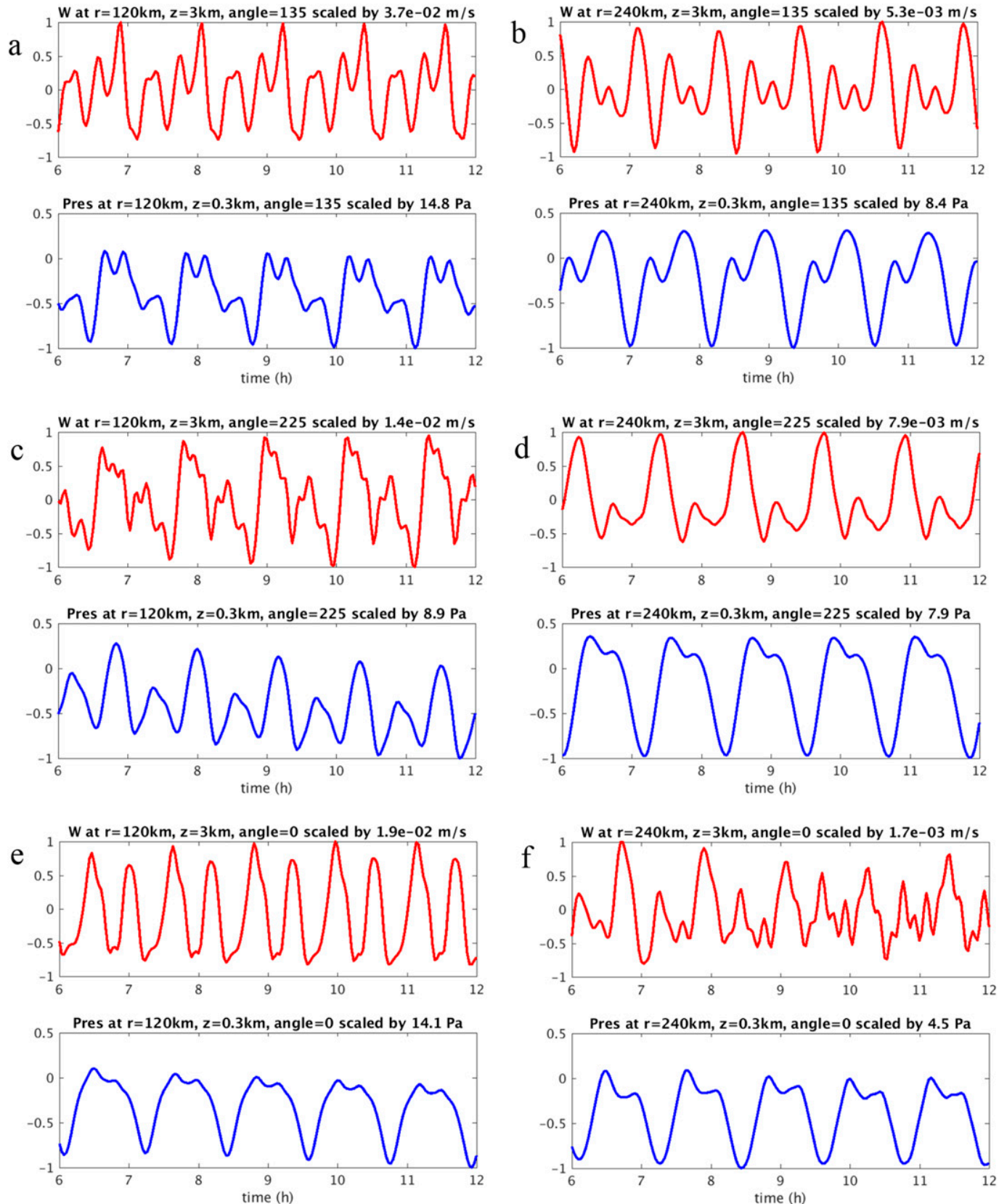

FIG. 11. Time series from fixed points relative to the vortex center; each panel pair shows $w$ at $z=3 \mathrm{~km}$ and $p$ at $z=0.3 \mathrm{~km}$ : (a) $r=120 \mathrm{~km}, \lambda=135^{\circ}$; (b) $r=240 \mathrm{~km}, \lambda=135^{\circ}$; (c) $r=120 \mathrm{~km}, \lambda=225^{\circ}$; (d) $r=240 \mathrm{~km}$, $\lambda=225^{\circ}$; (e) $r=120 \mathrm{~km}, \lambda=0^{\circ}$; (f) $r=240 \mathrm{~km}, \lambda=0^{\circ}$. Each time series has been scaled by its extreme value indicated on each plot.

We consider results from two additional simulations, with $V_{m}=40 \mathrm{~m} \mathrm{~s}^{-1}$ and with $V_{m}=60 \mathrm{~m} \mathrm{~s}^{-1}$. The radial and vertical structure of the wind field and the background stratification are the same. Different strengths of the warm core anomalies associated with stronger or weaker vortices do lead to some changes in stratification in the inner core, but the differences in the wave radiation region are insignificant. However, following the same procedure for defining the periods and durations of the pulsation results in different values for those parameters. For $V_{m}=40 \mathrm{~m} \mathrm{~s}^{-1}, t_{i}=1.46 \mathrm{~h}$ and $\tau_{f}=0.49 \mathrm{~h}$; for $V_{m}=$ $60 \mathrm{~m} \mathrm{~s}^{-1}, t_{i}=0.98 \mathrm{~h}$ and $\tau_{f}=0.33 \mathrm{~h}$.

One might expect that the resulting wave fields would be nearly identical to those shown above, but for a 
rescaling of time. This is not quite correct. Although the rotation speed of the heat source and the advection by the tangential wind are equally modified the outward radiation speeds remain about the same. Thus, the relative azimuthal and radial propagations of the waves are different. Figure 12a shows $w$ at $z=6 \mathrm{~km}$ at $t=3.13 \mathrm{~h}$ after a single pulse for $V_{m}=40 \mathrm{~m} \mathrm{~s}^{-1}$, which is equivalent to $t=2.5 \mathrm{~h}$ for $V_{m}=50 \mathrm{~m} \mathrm{~s}^{-1}$, as shown in Fig. $4 \mathrm{~d}$. The structures are similar, but for $V_{m}=40 \mathrm{~m} \mathrm{~s}^{-1}$ the waves have propagated further radially as compared to Fig. 4c. For $V_{m}=60 \mathrm{~m} \mathrm{~s}^{-1}$, at $t=2.1 \mathrm{~h}$, the waves have not traveled as far. Also, the wave amplitudes are greater and the radial wavelengths are shorter for larger $V_{m}$, both of which are due to the shorter forcing periods $\tau_{f}$ for each case.

Nonetheless, the dominant signal in the radiating waves comes from the forcing interval, and this can be seen clearly in the time series at fixed points. Figure 12 also shows time series for these two cases at $\lambda=135^{\circ}, r=$ $240 \mathrm{~km}$ (to emphasize the fast waves) and $\lambda=225^{\circ}, r=$ $120 \mathrm{~km}$ (to emphasize the short waves). In each $6 \mathrm{~h}$ period, there are about 4 dominant peaks for $V_{m}=$ $40 \mathrm{~m} \mathrm{~s}^{-1}$ and 6 dominant peaks for $V_{m}=60 \mathrm{~m} \mathrm{~s}^{-1}$. Higher-frequency waves are more predominant closer to the center and farther downwind in the tangential direction, as suggested in previous results as in Figs. 4 and 10.

\section{b. Rotational parameters}

In addition to the TC intensity, the pulsation period is controlled by two other parameters: the number of convective asymmetries and their rotation speeds relative to the tangential flow. A simple analysis of the model output discussed in section 2 suggested that the asymmetries were rotating at about $3 / 4$ of the peak rotation speed in the eyewall. In contrast, the classic theory of edge waves on a Rankine vortex predicts that $n=$ 2 asymmetries should rotate at only half the speed of the tangential flow (Lamb 1932). For more realistic vortices, many factors such as the smoothness of the vorticity profile with radius, the finite radial size of the vorticity anomalies, and the decrease with height of the tangential wind cause the asymmetries to retrograde against the mean flow less severely than in the classic result. For example, the $n=2$ unstable mode diagnosed in a hurricane-like vortex with $V_{\max }=36 \mathrm{~m} \mathrm{~s}^{-1}$ by Nolan and Montgomery (2002) had a rotational speed of $30 \mathrm{~m} \mathrm{~s}^{-1}$ at the RMW. Coupling to moist convection may also bring the asymmetries closer to the low-level tangential wind speeds.

Simulations with different rotation speeds of the convective asymmetries lead to accordingly modified frequencies of the $w$ and $p$ anomalies observed at fixed points. Some examples of this are shown in Fig. 13, again using $V_{m}=50 \mathrm{~m} \mathrm{~s}^{-1}$. If the asymmetries rotate at $1 / 2$ of the peak rotation rate, then the oscillation period is increased to $1.76 \mathrm{~h}$, and similarly, the dominant period of oscillations for both $p$ and $w$ is increased to the same value (Fig. 13a); if they rotate at the peak rate, the period is decreased to $0.88 \mathrm{~h}$, and the period of the response matches that as well (Fig. 13b).

Further complications arise if the primary convective asymmetry is characterized by a wavenumber other than $n=2$. Choosing 3 asymmetries means that 3 convective maxima will move through the preferred region during each vortex circulation time, changing the pulsation period to $0.78 \mathrm{~h}$, as shown in Fig. 13c. This result again uses the rotation rate of $3 / 4$ the peak rate, but we might expect that $n=3$ convective asymmetries would move even more closely with the mean flow, which would reduce the period further.

\section{c. Alternate forcing patterns}

Other forcing patterns may be relevant. For example, it may be possible in a very favorable, very low-shear environment that dynamical asymmetries would stimulate convective asymmetries that do not pulse in time, but that produce heat continuously. With this in mind, we changed the forcing function to consist of two anomalies of the form shown in (3.5) and (3.6), but on opposite sides of the vortex center, similar to the enhanced radar reflectivity that is frequently seen at the end points of TCs with elliptical eyewalls (Kuo et al. 1999; Reasor et al. 2000). The heat sources rotate at $3 / 4$ the peak speed, but they release heat continuously, without pulsing. In this case, the $n=0$ forcing is continuous and only drives a symmetric, direct overturning, so the results shown here only use the response summed from $n=2, n=4$, and for additional accuracy in this case, $n=6$; the odd wavenumbers are not excited.

The waves produced by continuously forced asymmetries have some interesting differences from the waves produced by pulsing. Once the response reaches steady state, the radiating wave fields as shown in Fig. 14 only rotate in time, with no structural evolution in radius or height. While the low-level response as shown in the nearsurface pressure field in Fig. 14a is almost entirely $n=2$, at higher altitude the response is predominantly $n=4$ in the inner core, transitioning to predominantly $n=2$ at larger distances (Fig. 14b). The vertical structure of the standing wave pattern (Fig. 14c) shows high-frequency waves with shorter radial wavelengths in the region from $r=$ 100 to $r=175 \mathrm{~km}$. Relative to a fixed azimuth, the phase lines of these waves propagate outward and downward as the wave field rotates cyclonically with the forcing.

The radiation pattern in the inner core suggests that waves are radiated outward and downward from the 

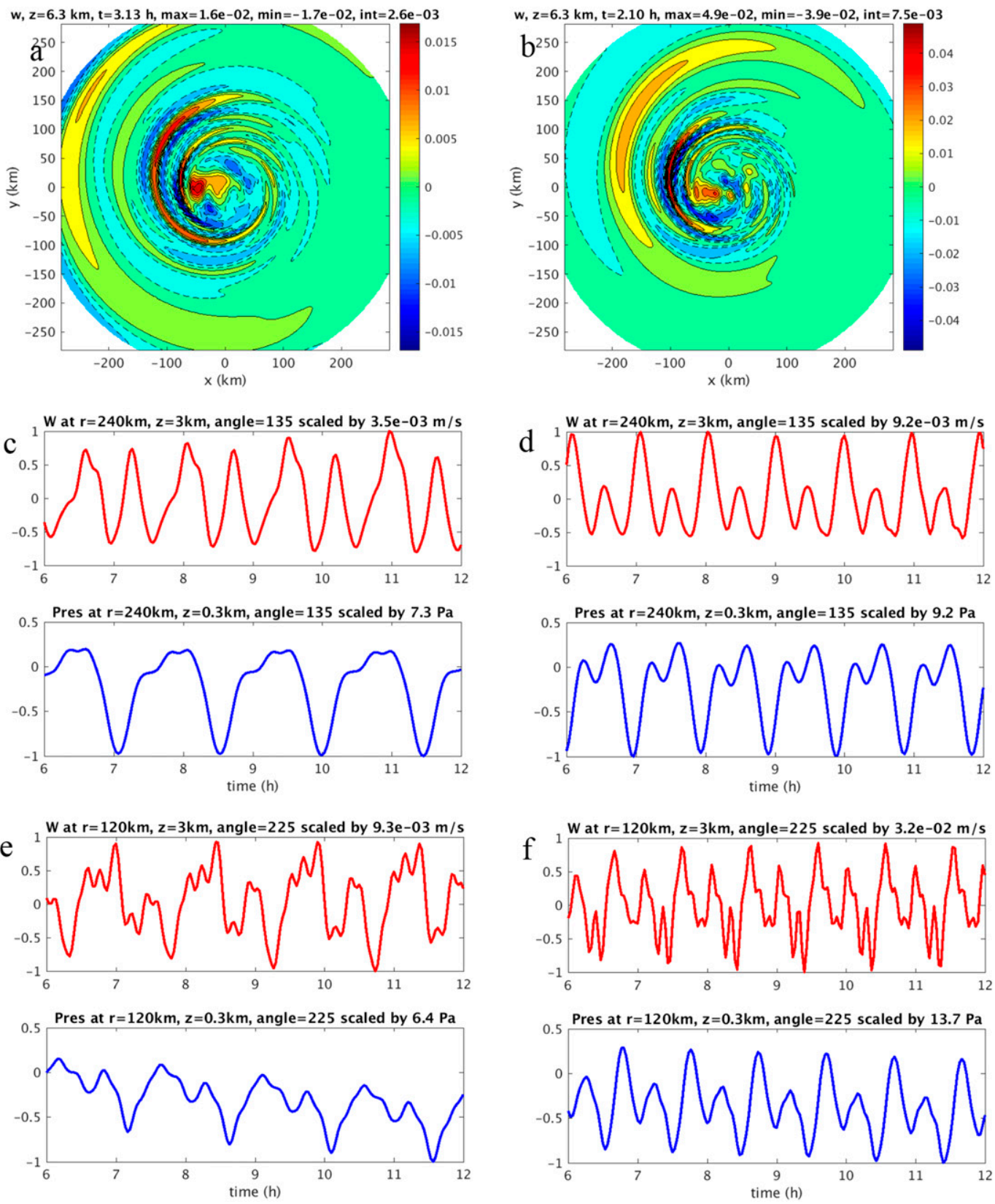

FIG. 12. Results for basic-state vortices of different strengths: (a) $w$ at $t=3.13 \mathrm{~h}$ for $V_{m}=40 \mathrm{~m} \mathrm{~s}^{-1}$; (b) $w$ at $t=$ $2.10 \mathrm{~h}$ for $V_{m}=60 \mathrm{~m} \mathrm{~s}^{-1}$; (c) for $V_{m}=40 \mathrm{~m} \mathrm{~s}^{-1}$, time series of $w$ and $p$ at $r=240 \mathrm{~km}, \lambda=135^{\circ}$; (d) for $V_{m}=60 \mathrm{~m} \mathrm{~s}^{-1}$, time series of $w$ and $p$ at $r=240 \mathrm{~km}, \lambda=135^{\circ}$; (e) for $V_{m}=40 \mathrm{~m} \mathrm{~s}^{-1}$, time series of $w$ and $p$ at $r=120 \mathrm{~km}, \lambda=225^{\circ}$; (f) for $V_{m}=40 \mathrm{~m} \mathrm{~s}^{-1}$, time series of $w$ and $p$ at $r=120 \mathrm{~km}, \lambda=135^{\circ}$.

heat sources at $r=50 \mathrm{~km}$, then reflect off the surface, with the shorter $n=4$ waves radiating upward at a steeper angle than the longer $n=2$ waves. This structure closely matches the structure of unstable modes that were identified by Hodyss and Nolan (2008) in hurricanelike, baroclinic vortices with sufficiently sharp negative vorticity gradients near the RMW. These instabilities are vertically varying versions of the coupled vortex and gravity wave instabilities discovered for isolated vortices in shallow-water equations or strongly stratified fluids (Ford 1994; Plougonven and Zeitlin 2002; Schecter and Montgomery 2004; Billant and Les Dizès 2009; Menelaou and Yau 2018). The relationship between the forced, radiating response shown here and coupled vortex-gravity wave modes is further discussed in appendix B. 

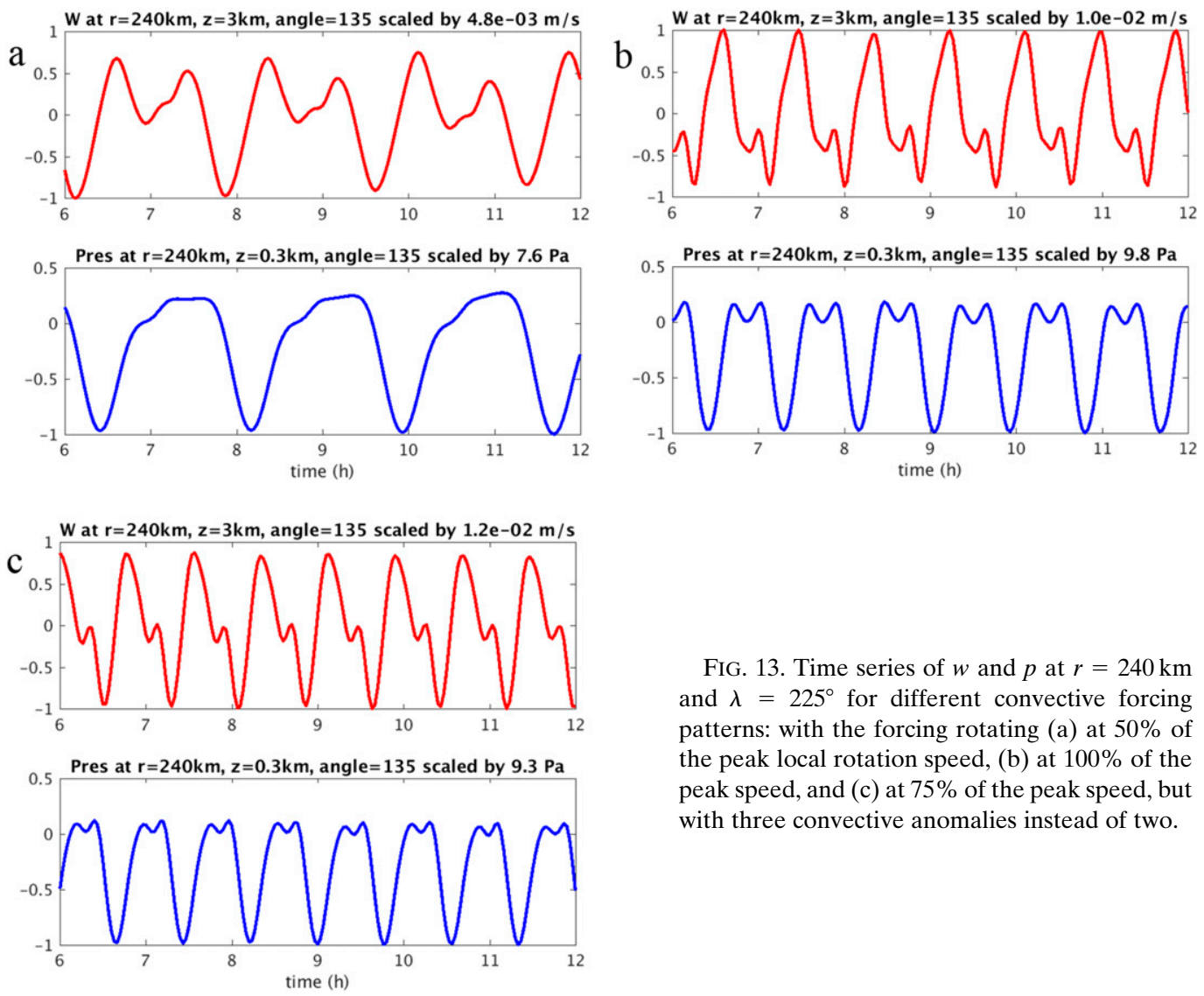

FIG. 13. Time series of $w$ and $p$ at $r=240 \mathrm{~km}$ and $\lambda=225^{\circ}$ for different convective forcing patterns: with the forcing rotating (a) at $50 \%$ of the peak local rotation speed, (b) at $100 \%$ of the peak speed, and (c) at $75 \%$ of the peak speed, but with three convective anomalies instead of two.

The response to three rotating, continuously forced asymmetries was quite similar, except that the horizontal structure showed only $n=3$ asymmetries and little apparent expression of other wavenumbers (not shown). The reason why $n=4$ appears for the previous case is that due to the small sizes of the two localized heat sources in comparison to the circumference of the inner core, they are not well-represented by $n=2$ alone, and $n=4$ is required to accurately represent the variation of the source function around the circle. For three heat sources, the distance between each heat source is nearly equal to the sizes of the sources themselves, so they have very little projection onto wavenumbers other than $n=3$.

We also considered the response to a heat source that is pulsing, but not rotating. Here we use the same frequency and duration of the pulses as in section $3 \mathrm{c}$, but the heat source remains fixed $50 \mathrm{~km}$ east of the vortex center. As shown in Fig. 14d, this produces a radiating wave pattern that is somewhat different in that it lacks the distinct "spiral" quality that is seen in all of the previous results or in NZ17. However, time series of $p$ and $w$ are similar to those produced by rotating heat sources, dominated at the surface by the pulsation

frequency, and showing signals from both short and long waves in $w$ (not shown).

\section{d. Stratification}

The environmental stratification in our linear model is defined by the temperature profile of the Jordan (1958) sounding. As shown in Fig. 2c, the squared BruntVäisälä frequency $N^{2}$ ranges from about $0.7 \times 10^{-4}$ to $1.2 \times 10^{-4} \mathrm{~s}^{-2}$ below $13 \mathrm{~km}$, and then increases to over $6.0 \times 10^{-4} \mathrm{~s}^{-2}$ in the lower stratosphere. To assess the effects of tropospheric $N^{2}$ on the wave radiation, we constructed soundings with $N^{2}$ set to values of $0.5,1.0$, and $2.0 \times 10^{-4} \mathrm{~s}^{-2}$ in the troposphere, and $5.0 \times 10^{-4} \mathrm{~s}^{-2}$ in the stratosphere. For a given lower boundary temperature $T_{0}$, a temperature profile that provides a constant stratification $N_{c}^{2}$ is prescribed by the following formula:

$$
T(z)=T_{0} e^{-N_{c}^{2} z / g}+\frac{g^{2}}{c_{p} N_{c}^{2}}\left(1-e^{-N_{c}^{2} z / g}\right),
$$

where $c_{p}$ is the heat capacity of dry air at constant pressure and $g$ is the gravitational acceleration. With $T_{0}=300 \mathrm{~K}$ and each value for $N_{c}^{2}$, this formula is used 

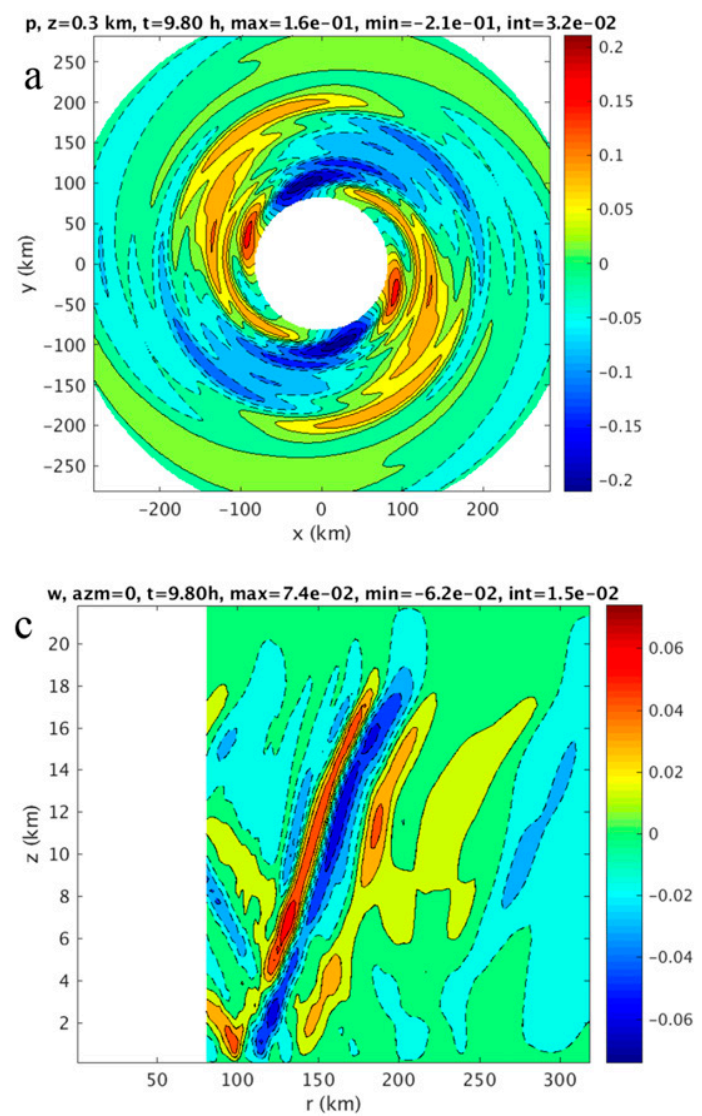
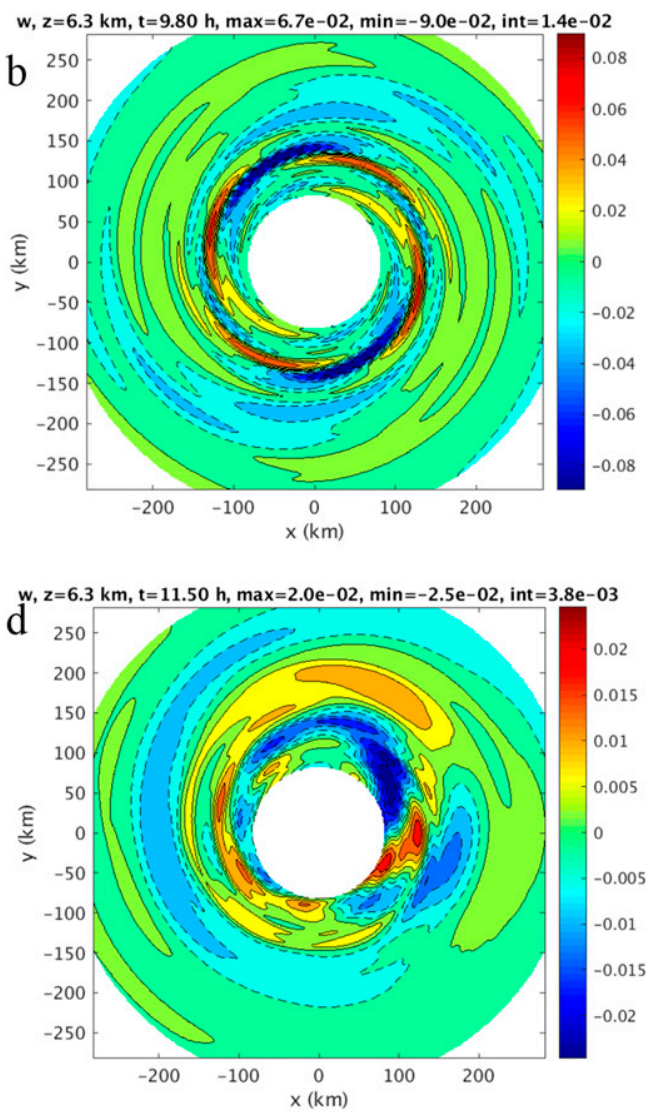

FIG. 14. Results for alternate forcing functions: (a) for two steady heat sources, $p$ at $0.3 \mathrm{~km}$; (b) $w$ at $6.3 \mathrm{~km}$; (c) radius-height section of $w$ along $\lambda=0^{\circ}$; (d) for a stationary but pulsing heat source, $w$ at $6.3 \mathrm{~km}$.

to compute $T(z)$ from $z=0$ to $14.5 \mathrm{~km}$. $T_{0}$ is reset to the value at $14.5 \mathrm{~km}$, and then the formula with $N_{c}^{2}=5.0 \times 10^{-4} \mathrm{~s}^{-2}$ is used for $T$ above $14.5 \mathrm{~km}$. The values of $T$ at the three grid levels around $14.5 \mathrm{~km}$ are averaged with their neighbors three times to provide a smooth transition between the troposphere and stratosphere. For $N_{c}^{2}=1.0 \times 10^{-4} \mathrm{~s}^{-2}, N^{2}(r, z)$ that results after balancing the vortex is shown in Fig. 2 d.

For the control-case rotational parameters, singlepulse and repeated-pulse simulations were performed for $N_{c}^{2}=0.5,1.0$, and $2.0 \times 10^{-4} \mathrm{~s}^{-2}$. The results for $N_{c}^{2}=1.0 \times 10^{-4} \mathrm{~s}^{-2}$ were nearly identical to those with the Jordan sounding and are not shown, but Hovmöller diagrams for the other values are shown in Fig. 15. As indicated by the dispersion relation (4.3), changes in $N^{2}$ cause changes in the phase speeds of the waves. Comparing to the previous results in Fig. 8, the amplitudes of the $w$ and $p$ waves are a little more than twice as large for $0.5 \times 10^{-4} \mathrm{~s}^{-2}$, and less than half as large for $2.0 \times 10^{-4} \mathrm{~s}^{-2}$ (note the changes in color ranges and contour values between the various plots in Figs. 8 and 15).

Results from the previous section showed that the period of the convective forcing determines the frequencies of the locally observed oscillations. Despite the large changes in the phase speeds of the waves, time series at fixed points for the differing values of $N^{2}$ found that this is still the case: the dominant periods are still equal to the forcing period of $1.17 \mathrm{~h}$ (not shown). Furthermore, differences in the tropospheric stratification are relatively small in the tropics, so variations in environmental stability can be expected to have virtually no effect on the relationship between TC intensity and point observations of wind or pressure perturbations.

\section{Comparison of the waves in the linear model, WRF Model, and observations}

As the convective forcing used in the calculations above was inspired by what is produced in an idealized WRF simulation of a tropical cyclone, we should consider how well they match the gravity wave patterns in the same simulation. The raw output of the idealized simulation is shown in Fig. 1a, and also in Fig. 2a of NZ17. There are some strong similarities between those figures and the spiral patterns produced by rotating and pulsing convective forcing as shown in Fig. 10 above. 

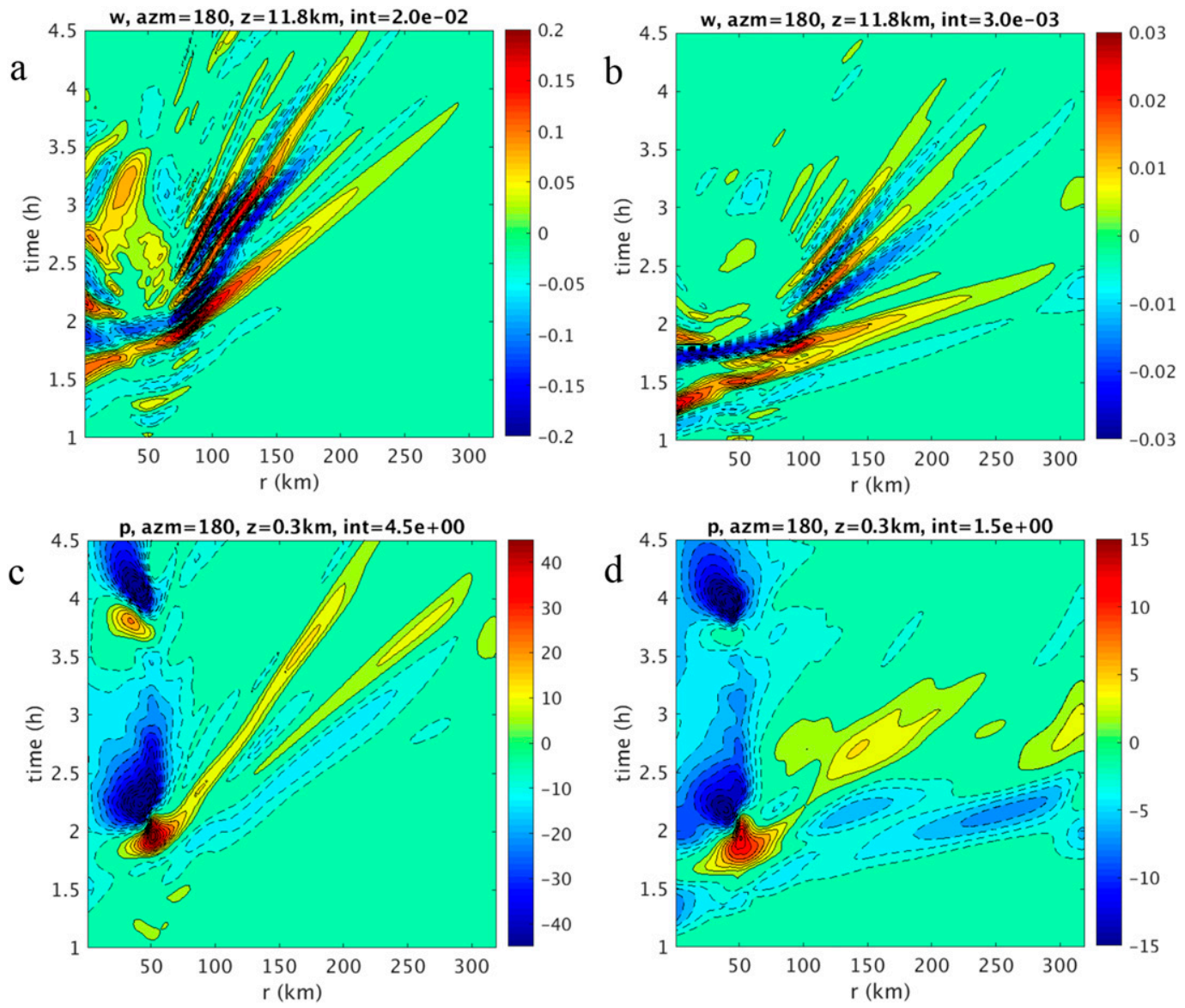

FIG. 15. Results for different stratifications: (a) for $N^{2}=5.0 \times 10^{-5} \mathrm{~s}^{-2}$, Hovmöller diagram of $w$ at $z=11.8 \mathrm{~km}$ along $\lambda=180^{\circ}$; (b) for $N^{2}=2.0 \times 10^{-4} \mathrm{~s}^{-2}$, Hovmöller diagram of $w$ at $z=11.8 \mathrm{~km}$ along $\lambda=180^{\circ}$; (c) for $N^{2}=$ $5.0 \times 10^{-5} \mathrm{~s}^{-2}$, Hovmöller diagram of $p$ at $z=0.3$ along $\lambda=180^{\circ}$; (d) for $N^{2}=2.0 \times 10^{-4} \mathrm{~s}^{-2}$, Hovmöller diagram of $p$ at $z=0.3 \mathrm{~km}$ along $\lambda=180^{\circ}$.

However, the waves produced by our linear model, with only $2 \mathrm{~km}$ grid spacing and substantial diffusion $(\nu=$ $20 \mathrm{~m}^{2} \mathrm{~s}^{-1}$ ), are obviously smoother and have significantly longer radial wavelengths. A closer comparison can be made by filtering the model output to contain only the same azimuthal wavenumbers as in 3DVPAS. The $w$ field after filtering to include only $n=2$ to 5 can be seen in Fig. 3 of NZ17. Here we show in Fig. 16 two additional examples from the same simulation, but using $n=1$ to 5 , and in addition, the data has been rotated clockwise $90^{\circ}$ so that the downshear-left quadrant is in the same location as the peak of the convective forcing in 3DVPAS. These two snapshots were chosen arbitrarily simply to show the extent to which the filtered waves in the model can be similar to those of the linear model. Despite the azimuthal filtering, the model output still shows finer scales, especially at larger distances from the center, which is generally not seen in the linear simulations.
The differences between the WRF simulation and 3DVPAS are greater in the vertical structure of the $w$ field. The plots in the lower half of Fig. 16 show one of the more favorable comparisons, which is still not very good. In each case the vertical cross section shows vertical velocity on the opposite side of the vortex from the convectively active region, and the figure axis ranges and aspect ratio have been adjusted to be as similar as possible, except for the amplitudes. Again, the $1 \mathrm{~km}$ resolution WRF simulation shows far more detailed structure and is considerably noisier. This is because of its fully nonlinear dynamics, smaller grid spacing, and smaller horizontal and vertical diffusion. Still, there are some similarities: for example, both models show separate trains of $w$ maxima in the lower and upper tropospheres. While the outward sloping phase lines of $w$ are easily seen in 3DVPAS, one can see evidence of this in the WRF simulation, for example from about $y=160 \mathrm{~km}, z=4 \mathrm{~km}$ to $y=200 \mathrm{~km}$, 

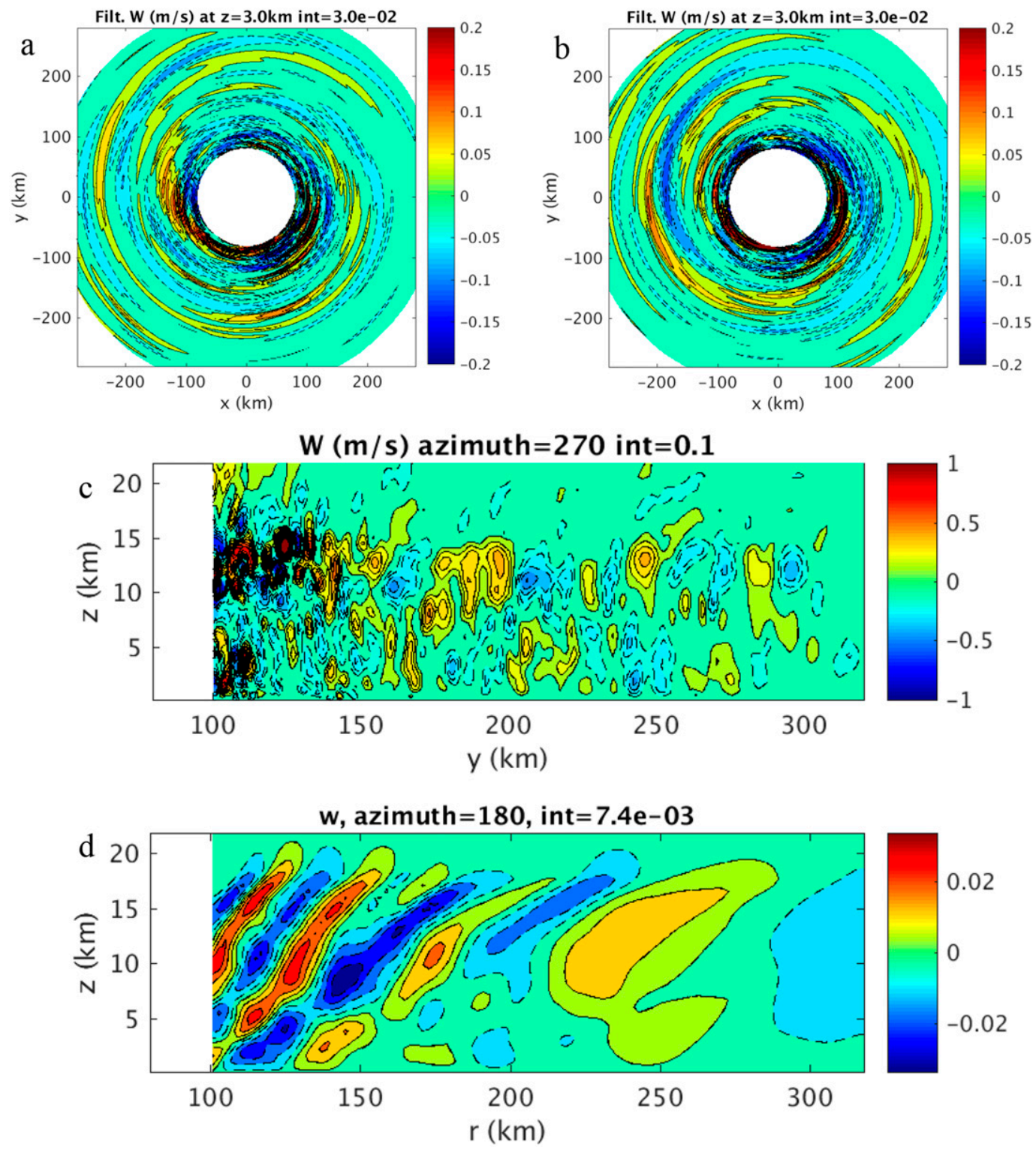

FIG. 16. Vertical velocity fields from the idealized simulation of NZ17, and comparison to the linear model: (a) filtered $w$ field near $z=3.0 \mathrm{~km}$ from the simulation at an arbitrarily selected time; (b) as in (a), but from a different time; (c) vertical slice of $w$ at azimuth angle $270^{\circ}$ from east at an arbitrary time; (d) vertical slice of $w$ from the linear model control case with repeating forcing at $t=12.0 \mathrm{~h}$.

$z=12 \mathrm{~km}$, with stronger and smaller-scale $w$ perturbations lying along the axis of this feature. Another, less organized outward-sloping axis of upward motion can be seen from $y=200 \mathrm{~km}$ to $y=250 \mathrm{~km}$. Another significant difference, also seen in the horizontal plots, is the persistence of smaller-scale anomalies out to larger radius, especially at higher altitude. This could be due to the significantly lower diffusion in the nonlinear simulation, but could also be due to the radial outflow in the upper troposphere, which is not present in 3DVPAS.
To date, direct observations of the spiral gravity waves are limited to what was presented in NZ17. From aircraft data they inferred that the smaller-scale $w$ waves at flight level had radial wavelengths of $4-6 \mathrm{~km}$ and radial phase speeds of $20-25 \mathrm{~m} \mathrm{~s}^{-1}$. In the idealized WRF simulations, the wavelength of these waves is typically about twice as large but they have about the same phase speed, whereas in our linear model, the wavelengths of the "secondary waves" range from 10 to $30 \mathrm{~km}$ (e.g., Figs. 4, 5, and 7). The fixed-point observations of surface pressure in NZ17 indicate dominant periods of about 
$1 \mathrm{~h}$, but unlike for the $w$ waves, the wavelengths and phase speeds cannot be estimated separately. Nonetheless, these appear to be equivalent to the deeper, broader, and faster "primary waves" revealed in 3DVPAS.

\section{Conclusions}

This paper has sought to determine whether the outward-propagating spiral gravity waves that can be seen in both observations and numerical simulations of TCs can be attributed to particular features of the TC itself. Based on inspection of the asymmetric pattern of moist diabatic heating in an idealized TC simulation, we considered whether the relevant convective forcing was the pulsation of convective asymmetries as they move through the convectively active region in the downshearleft quadrant of a weakly sheared TC, as had been seen before in previous studies (Black et al. 2002; Braun et al. 2006; Braun and Wu 2007; Reasor et al. 2009). Analytical functions were used to construct rotating heat sources that turned on and off as they entered one quadrant of a tropical cyclone-like vortex. A linear, nonhydrostatic model of perturbations to a balanced vortex (3DVPAS) was used to simulate the gravity waves generated by this rotating and pulsing forcing.

The gravity waves produced by this forcing are found to have many similarities to spiral gravity waves seen in the observations and numerical simulations of NZ17. With some similarity to what has been shown for transient heat sources in resting atmospheres (Nicholls et al. 1991; Mapes 1993; Alexander and Holton 2004), each pulse of heating produces two types of waves. The primary wave has longer radial wavelength, extends the depth of the troposphere, and has an outward phase speed of $60-70 \mathrm{~m} \mathrm{~s}^{-1}$. The primary wave has a distinct signal in the surface pressure. This wave is followed by a series of secondary waves with shorter radial and vertical wavelengths and slower phase speeds from 10 to $20 \mathrm{~m} \mathrm{~s}^{-1}$. By comparison to the waves seen in observations and simulations, the primary wave appears to be responsible for the low-frequency oscillations in surface pressure, and the secondary waves produce the shorter-wavelength oscillations in $w$ that are evident in the lower troposphere and also in the outflow of the TC. Due to the cyclonic advection of the tangential wind, the wave energies of both the primary and secondary waves predominantly radiate outward on the opposite side of the TC from the forcing.

We find that the periods of the $p$ and $w$ oscillations at fixed points are closely related to the frequency of the forcing. Typically, the $p$ oscillation period matches exactly the period of the forcing, although sometimes there is a secondary oscillation of weaker amplitude. The $w$ oscillations also show the primary frequency, but sometimes have two or three peaks in each cycle that are associated with the slower-moving secondary waves.

NZ17 speculated it might be possible to use observations of spiral gravity waves to infer TC intensity. The results here argue both for and against this possibility. A strong connection has been shown between the observed oscillation period at locations outside the TC and the frequency of the inner-core pulsation. On the other hand, considerable additional information is needed to infer the peak tangential wind speed in the eyewall. We would need to know the size of the RMW, the wavenumber of the dominant asymmetry, and the rotation speed of that asymmetry relative to the peak tangential flow. It could be possible to estimate some of this data from satellite observations, but of course satellite images that provide information of this detail can also provide good estimates of intensity from completely different methods.

Regardless of their potential utility for estimating TC intensity, spiral gravity waves radiating from TCs are interesting, and beautiful. Further comparisons between observed and simulated waves may provide guidance on how to improve the representation of inner-core dynamical processes in numerical models. More analysis of in situ observations of the passing waves, both aloft and at the surface, may reveal additional features that can be related inner-core processes.

Acknowledgments. The WRF simulations used in this study were produced at the University of Miami Center for Computational Sciences. The output from these simulations are archived and available and can be remotely transferred. D. Nolan was supported by the National Science Foundation under Award AGS1654831. Comments on earlier versions of the manuscript by R. Evans, J. Ge, J. Hlywiak, J. Zhang, T. Dunkerton, and two anonymous reviewers helped to improve the paper.

\section{APPENDIX A}

\section{Influence of the Model Ceiling at $22 \mathrm{~km}$}

The gravity waves simulated by our model propagate upward as well as outward, and reach the upper-level damping layer and the model top within $2 \mathrm{~h}$ of being generated, and before they have radiated $100 \mathrm{~km}$ outward (see, e.g., Figs. 5, 9). Given the close proximity of the model top and the damping layer, it is important to consider whether they have a significant effect. 

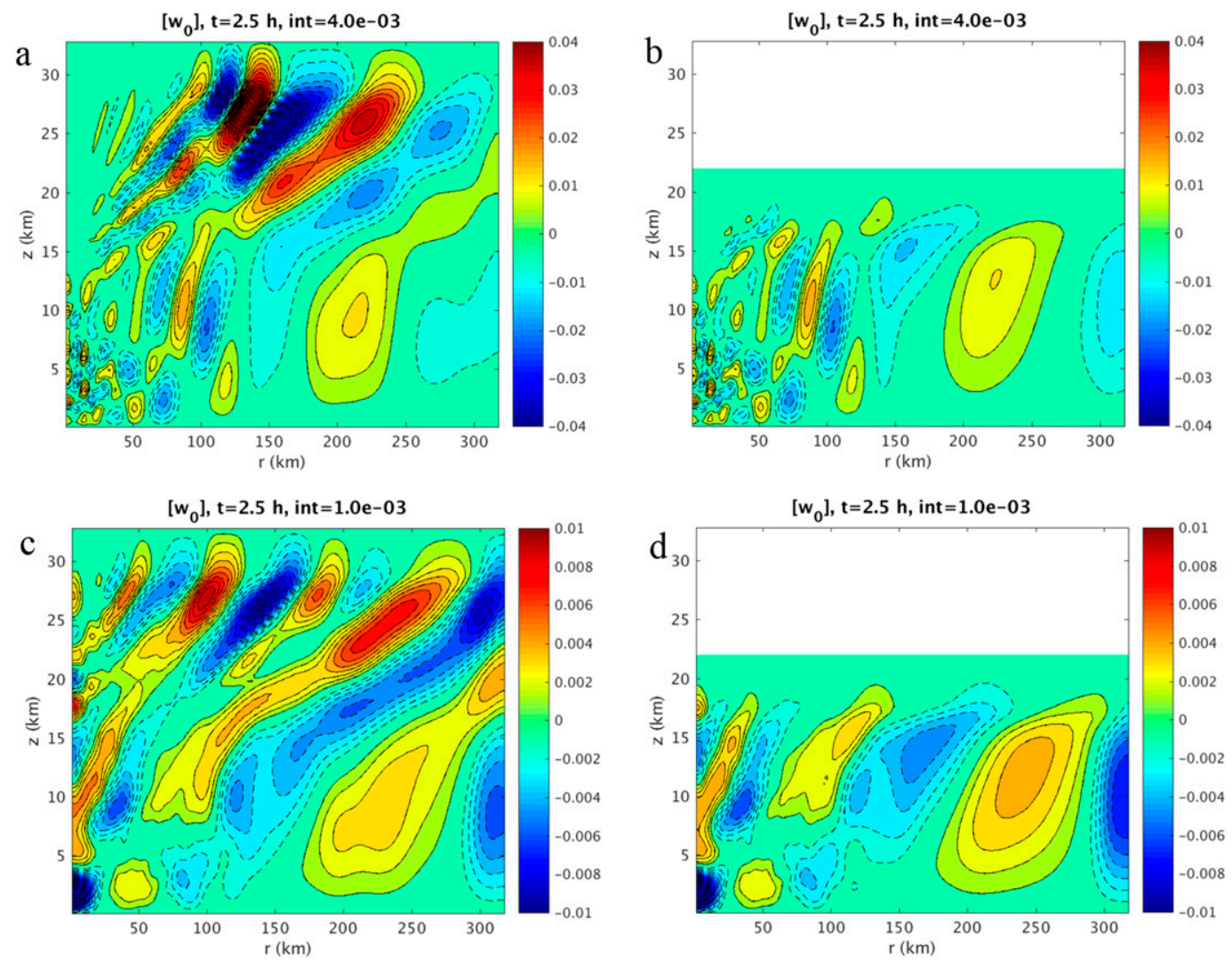

FIG. A1. Comparison of results for symmetric motions with the model top moved to $z=33 \mathrm{~km}$ : (a) $w$ field generated at $t=2.5 \mathrm{~h}$ for a single pulse in a resting atmosphere with the model top at $33 \mathrm{~km}$; (b) as in (a), but with the model top at $22 \mathrm{~km}$; (c) single pulse in the control case TC with the model top at $33 \mathrm{~km}$; (d) as in (c), but with the model top at $22 \mathrm{~km}$. Units are $\mathrm{m} \mathrm{s}^{-1}$.

To address this concern, we show the results of two additional simulations where the model top has been elevated to $33 \mathrm{~km}$. The number of vertical levels is increased to 60 so that the vertical grid spacing is the same as used in the body of the paper, and the vertical damping layer has the same depth of $4 \mathrm{~km}$. We consider the symmetric response to both the isolated pulsed in a resting atmosphere (as described in section $4 \mathrm{~b}$ ) and the symmetric control-case pulse in the control-case TC (as described in section 3b).

The symmetric $w$ fields at $t=2.5 \mathrm{~h}$ for these two simulations are compared in Fig. A1 to results with the model top at $22 \mathrm{~km}$. It is evident that with the higher top, the waves continue to propagate upward and outward, with $w$ increasing with height due to the decreasing density. The panels are shown with the same aspect ratio and the same contour intervals. Comparing the panels on the left and right, we find that the $w$ fields in the troposphere with the model top at $33 \mathrm{~km}$ are quite similar to those with the top at $22 \mathrm{~km}$. Below $15 \mathrm{~km}$ and inside $r=150 \mathrm{~km}$, they are nearly identical. Some differences are visible at larger radii, which might be expected because these motions of the faster, deeper waves are somewhat affected by the upper-level boundary condition.

\section{APPENDIX B}

\section{Stability of the Basic-State Vortex}

A modified Rankine vortex has constant vorticity in its core, which drops sharply to a much lower value outside the RMW, and then decays slowly with radius. As such, it does not meet the usual necessary condition for asymmetric instability of having a change in sign of the vorticity gradient (Michalke and Timme 1967; Schubert et al. 1999). In a stratified fluid, a Rankine or modified Rankine vortex can also be unstable to asymmetric modes that involve coupling between edge waves on the high-vorticity core and spiral gravity waves (Ford 1994; Plougonven and Zeitlin 2002; Schecter and Montgomery 2004; Hendricks et al. 2010). However, if the vortex decays with height, the potential for such instabilities is substantially mitigated (Hodyss and Nolan 2008). 

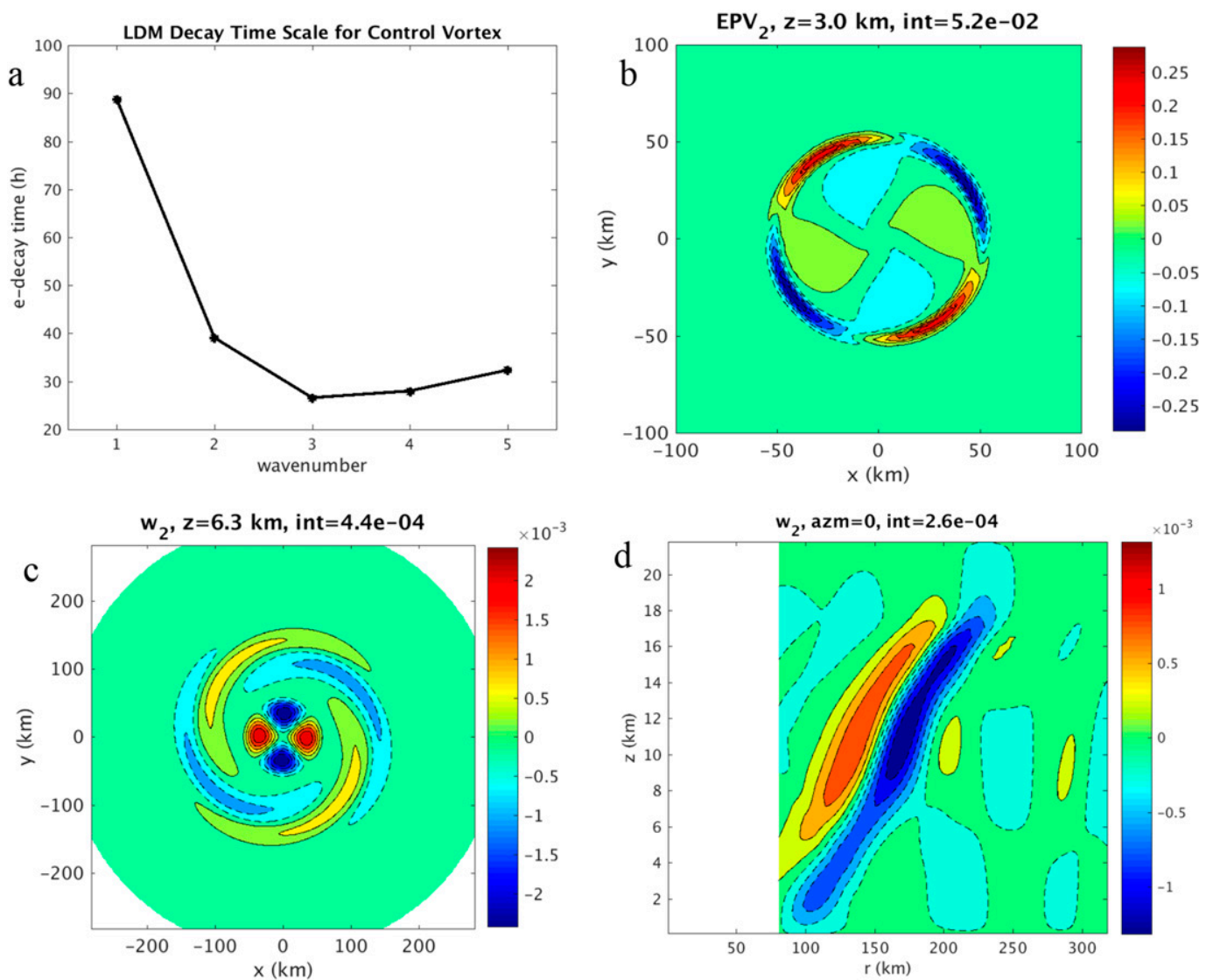

FIG. B1. Stability and least damped modes of the control-case vortex: (a) exponential-decay time scales for the slowest decaying modes for $n=1$ to 5; (b) perturbation Ertel's potential vorticity (PVU; 1 PVU = $10^{-6} \mathrm{~K} \mathrm{~kg}^{-1} \mathrm{~m}^{2} \mathrm{~s}^{-1}$ ) at $z=3 \mathrm{~km}$ for the $n=2$ mode (note smaller axis ranges); (c) $w$ at $z=6.3 \mathrm{~km}$ for $n=2$; (d) radius-height cross section of $w$ with data inside $80 \mathrm{~km}$ suppressed. The mode amplitude is normalized to have maximum perturbation radial velocity of $1 \mathrm{~m} \mathrm{~s}^{-1}$.

A stable vortex may still support slowly decaying asymmetric modes, and if the vortex is forced with similar structures and frequencies, the response will be amplified, as found by Moon and Nolan (2010). Using the same methods as in Nolan and Montgomery (2002) and Hodyss and Nolan (2008), the modes with the largest growth rates were found for $n=1$ to 5 . The growth rates for all five modes are negative, and these are plotted in terms of $e$-decay times in Fig. B1.

The horizontal and vertical structures for these five modes are quite similar, and are shown for $n=2$. Each mode consists of an edge wave in perturbation potential vorticity at low levels coupled to an outward-slanted gravity wave. The modes rotate at nearly the peak rotation speed of the vortex, increasing from $46 \mathrm{~m} \mathrm{~s}^{-1}$ for $n=1$ to $48 \mathrm{~m} \mathrm{~s}^{-1}$ for $n=5$. Thus, we can expect that when there is continuous forcing of a rotating asymmetry in the inner core, with rotation speeds comparable to the peak rotation rate, there will be some resonance and the dynamical response will be similar to the structures shown in Fig. B1.

\section{REFERENCES}

Alexander, M. J., and J. R. Holton, 2004: On the spectrum of vertically propagating gravity waves generated by a transient heat source. Atmos. Chem. Phys., 4, 923-932, https://doi.org/ 10.5194/acp-4-923-2004.

_, — , and D. R. Durran, 1995: The gravity wave response above deep convection in a squall line simulation. J. Atmos. Sci., 52, 2212-2226, https://doi.org/10.1175/1520-0469(1995) 052<2212:TGWRAD $>2.0 . \mathrm{CO} ; 2$.

Anthes, R. A., 1972: Development of asymmetries in a threedimensional numerical model of the tropical cyclone. Mon. Wea. Rev., 100, 461-476, https://doi.org/10.1175/1520-0493(1972) 100<0461:DOAIAT $>2.3$.CO;2.

Billant, P., and S. Les Dizès, 2009: Waves on a columnar vortex in a strongly stratified fluid. Phys. Fluids, 21, 106602, https:// doi.org/10.1063/1.3248366.

Black, M. L., R. W. Burpee, and F. D. Marks Jr., 1996: Vertical motion characteristics of tropical cyclones determined with airborne Doppler radar radial velocities. J. Atmos. Sci., 53, 
1887-1909, https://doi.org/10.1175/1520-0469(1996)053<1887: VMCOTC $>2.0 . \mathrm{CO} ; 2$.

- J. F. Gamache, F. D. Marks, C. E. Samsury, and H. E. Willoughby, 2002: Eastern Pacific Hurricanes Jimena of 1991 and Olivia of 1994: The effect of vertical shear on structure and intensity. Mon. Wea. Rev., 130, 2291-2312, https://doi.org/ 10.1175/1520-0493(2002)130<2291:EPHJOA > 2.0.CO;2.

Braun, S. A., and L. Wu, 2007: A numerical study of Hurricane Erin (2001). Part II: Shear and the organization of eyewall vertical motion. Mon. Wea. Rev., 135, 1179-1194, https:// doi.org/10.1175/MWR3336.1.

— M. T. Montgomery, and Z. Pu, 2006: High-resolution simulation of Hurricane Bonnie (1998). Part I: The organization of eyewall vertical motion. J. Atmos. Sci., 63, 19-42, https:// doi.org/10.1175/JAS3598.1.

Chane Ming, F., Z. Chen, and F. Roux, 2010: Analysis of gravitywaves produced by intense tropical cyclones. Ann. Geophys., 28, 531-547, https://doi.org/10.5194/angeo-28-531-2010.

— - S. Jolivet, Y.-A. Liou, F. Jegou, D. Mekies, and J.-S. Hong, 2019: Elliptical structures of gravity waves produced by Typhoon Soudelor in 2015 near Taiwan. Atmosphere, 10, 260, https://doi.org/10.3390/atmos10050260.

Corbosiero, K. L., and J. Molinari, 2002: The effects of vertical wind shear on the distribution of convection in tropical cyclones. Mon. Wea. Rev., 130, 2110-2123, https://doi.org/10.1175/15200493(2002)130<2110:TEOVWS > 2.0.CO;2.

Doyle, J. D., and Coauthors, 2017: A view of tropical cyclones from above: The Tropical Cyclone Intensity Experiment. Bull. Amer. Meteor. Soc., 98, 2113-2134, https://doi.org/10.1175/ BAMS-D-16-0055.1.

Dunion, J. P., 2011: Rewriting the climatology of the tropical North Atlantic and Caribbean Sea atmosphere. J. Climate, 24, 893908, https://doi.org/10.1175/2010JCLI3496.1.

Ford, R., 1994: The response of a rotating ellipse of uniform potential vorticity to gravity wave radiation. Phys. Fluids, 6, 3694-3704, https://doi.org/10.1063/1.868360.

Fovell, R. G., D. Durran, and J. R. Holton, 1992: Numerical simulations of convectively generated stratosphere gravity waves. J. Atmos. Sci., 49, 1427-1442, https://doi.org/10.1175/15200469(1992)049<1427:NSOCGS>2.0.CO;2.

Gall, R., J. Tuttle, and P. Hildebrand, 1998: Small-scale spiral bands observed in Hurricanes Andrew, Hugo, and Erin. Mon. Wea. Rev., 126, 1749-1766, https://doi.org/10.1175/ 1520-0493(1998)126<1749:SSSBOI >2.0.CO;2.

Guimond, S. R., G. M. Heymsfield, and F. J. Turk, 2010: Multiscale observations of Hurricane Dennis (2005): The effects of hot towers on rapid intensification. J. Atmos. Sci., 67, 633-654, https://doi.org/10.1175/2009JAS3119.1.

— M. A. Bourassa, and P. D. Reasor, 2011: A latent heat retrieval and its effects on the intensity and structure change of Hurricane Guillermo (1997). Part I: The algorithm and observations. J. Atmos. Sci., 68, 1549-1567, https://doi.org/10.1175/2011JAS3700.1.

Guinn, T. A., and W. H. Schubert, 1993: Hurricane spiral bands. J. Atmos. Sci., 50, 3380-3403, https://doi.org/10.1175/15200469(1993) $050<3380: \mathrm{HSB}>2.0 . \mathrm{CO} ; 2$.

Hendricks, E. A., W. H. Schubert, S. R. Fulton, and B. D. McNoldy, 2010: Spontaneous-adjustment emission of inertiagravity waves by unsteady vortical motion in the hurricane core. Quart. J. Roy. Meteor. Soc., 136, 537-548, https://doi.org/ 10.1002/QJ.547.

, B. D. McNoldy, and W. H. Schubert, 2012: Observed inner-core structural variability in Hurricane Dolly (2008). Mon. Wea. Rev., 140, 4066-4077, https://doi.org/10.1175/MWR-D-12-00018.1.
Hodyss, D., and D. S. Nolan, 2007: Linear anelastic equations for atmospheric vortices. J. Atmos. Sci., 64, 2947-2959, https:// doi.org/10.1175/JAS3991.1.

— and - 2008: The Rossby-inertia-buoyancy instability in baroclinic vortices. Phys. Fluids, 20, 096602, https://doi.org/ 10.1063/1.2980354.

Hoffmann, L., X. Wu, and M. J. Alexander, 2018: Satellite observations of stratospheric gravity waves associated with the intensification of tropical cyclones. Geophys. Res. Lett., 45, 1692-1700, https://doi.org/10.1002/2017GL076123.

Hong, S. Y., and J. O. J. Lim, 2006: The WRF single-moment 6-class microphysics scheme (WSM6). J. Korean Meteor. Soc, 42, 129-151.

—, Y. Noh, and J. Dudhia, 2006: A new vertical diffusion package with an explicit treatment of entrainment processes. Mon. Wea. Rev., 134, 2318-2341, https://doi.org/10.1175/ MWR3199.1.

Jordan, C. L., 1958: Mean soundings for the West Indies area. J. Meteor., 15, 91-97, https://doi.org/10.1175/1520-0469(1958) 015<0091:MSFTWI >2.0.CO;2.

Kim, S.-Y., and H.-Y. Chun, 2005: A numerical study of gravity waves induced by convection associated with Typhoon Rusa. Geophys. Res. Lett., 32, L24816, https://doi.org/10.1029/ 2005GL024662.

$\longrightarrow,-$, and D. L. Wu, 2009: A study on stratospheric gravity waves generated by Typhoon Ewiniar: Numerical simulations and satellite observations. J. Geophys. Res., 114, D22104, https://doi.org/10.1029/2009JD011971.

Kuester, M. A., M. J. Alexander, and E. A. Ray, 2008: A model study of gravity waves over Hurricane Humberto (2001). J. Atmos. Sci., 65, 3231-3246, https://doi.org/10.1175/2008JAS2372.1.

Kuo, H.-C., R. T. Williams, and J.-H. Chen, 1999: A possible mechanism for the eye rotation of Typhoon Herb. J. Atmos. Sci., 56, 1659-1673, https://doi.org/10.1175/1520-0469(1999) 056<1659:APMFTE $>2.0 . \mathrm{CO} ; 2$.

Kurihara, Y., and R. E. Tuleya, 1974: Structure of a tropical cyclone developed in a three-dimensional numerical simulation model. J. Atmos. Sci., 31, 893-919, https://doi.org/10.1175/ 1520-0469(1974)031<0893:SOATCD>2.0.CO;2.

Lamb, H., 1932: Hydrodynamics. Dover, 738 pp.

Lane, T. P., M. J. Reeder, and T. L. Clark, 2001: Numerical modeling of gravity wave generation by deep tropical convection. J. Atmos. Sci., 58, 1249-1274, https://doi.org/10.1175/15200469(2001)058<1249:NMOGWG > 2.0.CO;2.

Mallen, K. J., M. T. Montgomery, and B. Wang, 2005: Reexamining the near-core radial structure of the tropical cyclone primary circulation: Implications for vortex resiliency. J. Atmos. Sci., 62, 408-425, https://doi.org/10.1175/JAS-3377.1.

Mapes, B. E., 1993: Gregarious tropical convection. J. Atmos. Sci., 50, 2026-2037, https://doi.org/10.1175/1520-0469(1993)050<2026: GTC $>2.0 . \mathrm{CO} ; 2$.

Mathur, M. B., 1975: Development of a banded structure in a numerically simulated hurricane. J. Atmos. Sci., 32, 512-522, https://doi.org/10.1175/1520-0469(1975)032<0512: DOBSIA $>2.0 . \mathrm{CO} ; 2$.

Matsumoto, S., and H. Okamura, 1985: The internal gravity wave observed in Typhoon T8124 (Gay). J. Meteor. Soc. Japan, 63 37-51, https://doi.org/10.2151/jmsj1965.63.1_37.

Menelaou, K., and M. K. Yau, 2018: Spontaneous emission of spiral inertia-gravity waves and formation of elliptical eyewalls in tropical cyclone-like vortices: Three-dimensional nonlinear simulations. J. Atmos. Sci., 75, 2635-2658, https://doi.org/ 10.1175/JAS-D-17-0341.1. 
Michalke, A., and A. Timme, 1967: On the inviscid instability of certain two-dimensional vortex-type flows. J. Fluid Mech., 29, 647-666, https://doi.org/10.1017/S0022112067001090.

Montgomery, M. T., and R. J. Kallenbach, 1997: A theory for vortex Rossby waves and its application to spiral bands and intensity changes in hurricanes. Quart. J. Roy. Meteor. Soc., 123, 435-465, https://doi.org/10.1002/qj.49712353810.

Moon, Y., and D. S. Nolan, 2010: Do gravity waves transport angular momentum away from hurricanes? J. Atmos. Sci., 67, 117-135, https://doi.org/10.1175/2009JAS3088.1.

$\longrightarrow$, and _ 2015a: Spiral rainbands in a numerical simulation of Hurricane Bill (2009). Part I: Structures and comparisons to observations. J. Atmos. Sci., 72, 164-190, https://doi.org/ 10.1175/JAS-D-14-0058.1.

— and $\_$, 2015b: Spiral rainbands in a numerical simulation of Hurricane Bill (2009). Part II: Propagation of inner rainbands. J. Atmos. Sci., 72, 191-215, https://doi.org/10.1175/JASD-14-0056.1.

Nicholls, M. E., R. A. Pielke, and W. R. Cotton, 1991: Thermally forced gravity waves in an atmosphere at rest. J. Atmos. Sci. 48, 1869-1884, https://doi.org/10.1175/1520-0469(1991)048<1869: TFGWIA $>2.0 . \mathrm{CO} ; 2$.

Niranjan Kumar, K., T. K. Ramkumar, and M. Krishnaiah, 2011: MST radar observation of inertia-gravity waves generated from tropical cyclones. J. Atmos. Sol.-Terr. Phys., 73, 18901906, https://doi.org/10.1016/j.jastp.2011.04.026.

_ C. C. Kanaka Rao, A. Sandeep, and T. N. Rao, 2014: SODAR observations of inertia-gravity waves in the atmospheric boundary layer during the passage of tropical cyclone. Atmos. Sci. Lett., 15, 120-126, https://doi.org/10.1002/asl2.478.

Nolan, D. S., 2005: Instabilities in hurricane-like boundary layers. Dyn. Atmos. Oceans, 40, 209-236, https://doi.org/10.1016/ j.dynatmoce.2005.03.002.

__ 2011: Evaluating environmental favorableness for tropical cyclone development with the method of point-downscaling. J. Adv. Model. Earth Syst., 3, M08001, https://doi.org/10.1029/ 2011MS000063.

_ - and M. T. Montgomery, 2002: Nonhydrostatic, threedimensional perturbations to balanced, hurricane-like vortices. Part I: Linearized formulation, stability, and evolution. J. Atmos. Sci., 59, 2989-3020, https://doi.org/10.1175/15200469(2002)059<2989:NTDPTB > 2.0.CO;2.

—_ and L. D. Grasso, 2003: Nonhydrostatic, three-dimensional perturbations to balanced, hurricane-like vortices. Part II: Symmetric response and nonlinear simulations. J. Atmos. Sci., 60, 2717-2745, https://doi.org/10.1175/1520-0469(2003) $060<2717$ :NTPTBH $>2.0$. CO;2.

_ - and M. G. McGauley, 2012: Tropical cyclogenesis in wind shear: Climatological relationships and physical processes. Cyclones: Formation, Triggers, and Control, K. Oouchi and H. Fudeyasu, Eds., Nova Science Publishers, 1-34.

— , and J. A. Zhang, 2017: Spiral gravity waves radiating from tropical cyclones. Geophys. Res. Lett., 44, 3924-3931, https:// doi.org/10.1002/2017GL073572.

_- Y. Moon, and D. P. Stern, 2007: Tropical cyclone intensification from asymmetric convection: Energetics and efficiency. J. Atmos. Sci., 64, 3377-3405, https://doi.org/10.1175/JAS3988.1.
Pfister, L., and Coauthors, 1993: Gravity waves generated by a tropical cyclone during the STEP tropical field program: A case study. J. Geophys. Res., 98, 8611-8638, https://doi.org/10.1029/92JD01679.

Plougonven, R., and V. Zeitlin, 2002: Internal gravity wave emission from a pancake vortex: An example of wave-vortex interaction in strongly stratified flows. Phys. Fluids, 14, 1259-1268, https://doi.org/10.1063/1.1448297.

Reasor, P. D., M. T. Montgomery, F. D. Marks Jr., and J. F. Gamache, 2000: Low-wavenumber structure and evolution of the hurricane inner core observed by airborne dual-Doppler radar. Mon. Wea. Rev., 128, 1653-1680, https://doi.org/10.1175/ 1520-0493(2000)128<1653:LWSAEO > 2.0.CO;2.

— M. Eastin, and J. F. Gamache, 2009: Rapidly intensifying Hurricane Guillermo (1997). Part I: Low-wavenumber structure and evolution. Mon. Wea. Rev., 137, 603-631, https:// doi.org/10.1175/2008MWR2487.1.

_ R. F. Rogers, and S. Lorsolo, 2013: Environmental flow impacts on tropical cyclone structure diagnosed from airborne Doppler radar composites. Mon. Wea. Rev., 141, 2949-2969, https://doi.org/10.1175/MWR-D-12-00334.1.

Rogers, R., S. S. Chen, J. E. Tenerelli, and H. E. Willoughby, 2003: A numerical study of the impact of vertical shear on the distribution of rainfall in Hurricane Bonnie (1998). Mon. Wea. Rev., 131, 1577-1599, https://doi.org/10.1175//2546.1.

Sato, K., 1993: Small-scale wind disturbances observed by the MU radar during the passage of Typhoon Kelly. J. Atmos. Sci., 50, 518-537, https://doi.org/10.1175/1520-0469(1993)050<0518: SSWDOB $>2.0 . \mathrm{CO} ; 2$.

Schecter, D. A., 2008: The spontaneous imbalance of an atmospheric vortex at high Rossby number. J. Atmos. Sci., 65, 24982521, https://doi.org/10.1175/2007JAS2490.1.

— - and M. T. Montgomery, 2004: Damping and pumping of a vortex Rossby wave in a monotonic cyclone: Critical layer stirring versus inertia-buoyancy wave emission. Phys. Fluids, 16, 1334-1348, https://doi.org/10.1063/1.1651485.

Schubert, W. H., M. T. Montgomery, R. K. Taft, T. G. Guinn, S. R. Fulton, J. P. Kossin, and J. P. Edwards, 1999: Polygonal eyewalls, asymmetric eye contraction, and potential vorticity mixing in hurricanes. J. Atmos. Sci., 56, 1197-1223, https:// doi.org/10.1175/1520-0469(1999)056<1197:PEAECA >2.0.CO;2.

Senn, H. V., and H. W. Hiser, 1959: On the origin of hurricane spiral rain bands. J. Meteor., 16, 419-426, https://doi.org/ 10.1175/1520-0469(1959)016<0419:OTOOHS >2.0.CO;2.

Sutherland, B. R., 2010: Internal Gravity Waves. Cambridge University Press, $377 \mathrm{pp}$.

Tratt, D. M., and Coauthors, 2018: GHOST: A satellite mission concept for persistent monitoring of stratospheric gravity waves induced by severe storms. Bull. Amer. Meteor. Soc., 99, 1813-1828, https://doi.org/10.1175/BAMS-D-17-0064.1.

Vallis, G. K., 2006: Atmospheric and Oceanic Fluid Dynamics. Cambridge University Press, $745 \mathrm{pp}$.

Wang, Y., 2002: Vortex Rossby waves in a numerically simulated tropical cyclone. Part I: Overall structure, potential vorticity, and kinetic energy budgets. J. Atmos. Sci., 59, 1213-1238, https:// doi.org/10.1175/1520-0469(2002)059<1213:VRWIAN > 2.0.CO;2.

Wexler, H., 1947: Structure of hurricanes as determined by radar. Ann. N. Y. Acad. Sci., 48, 821-845, https://doi.org/10.1111/ j.1749-6632.1947.tb38495.x. 